CIEA-GR-01-02

ICN-UNAM-01/03

July 5, 2001

\title{
Area-Constrained Planar Elastica
}

\author{
Guillermo Arreaga, Riccardo Capovilla, \\ Departamento de Física \\ CINVESTAV IPN \\ Apdo. Postal 14740 \\ 07000 México, D.F., MEXICO \\ garreaga@fis.cinvestav.mx, capo@fis.cinvestav.mx
}

\section{Chryssomalis Chryssomalakos and Jemal Guven}

Instituto de Ciencias Nucleares

Universidad Nacional Autónoma de México

Apdo. Postal 70-543, 04510 México, D.F., MEXICO

chryss@nuclecu.unam.mx, jemal@nuclecu.unam.mx

\begin{abstract}
We determine the equilibria of a rigid loop in the plane, subject to the constraints of fixed length and fixed enclosed area. Rigidity is characterized by an energy functional quadratic in the curvature of the loop. We find that the area constraint gives rise to equilibria with remarkable geometrical properties: not only can the Euler-Lagrange equation be integrated to provide a quadrature for the curvature but, in addition, the embedding itself can be expressed as a local function of the curvature. The configuration space is shown to be essentially one-dimensional, with surprisingly rich structure. Distinct branches of integer-indexed equilibria exhibit self-intersections and bifurcations - a gallery of plots is provided to highlight these findings. Perturbations connecting equilibria are shown to satisfy a first order ODE which is readily solved. We also obtain analytical expressions for the energy as a function of the area in some limiting regimes.
\end{abstract}




\section{Contents}

\begin{tabular}{|lll}
\hline & Introduction & 2 \\
\hline
\end{tabular}

2 First Considerations 4

2.1 Energy functional . . . . . . . . . . . . . . . . . . . . . . . . . . . . . 4

2.2 Shape equation $\ldots \ldots \ldots \ldots$. . . . . . . . . . . . . . . . . . . . . . . . . . . . 5

2.3 Scaling . . . . . . . . . . . . . . . . . . . . . . . . . . 6

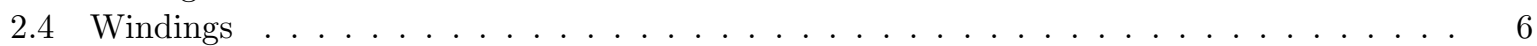

2.5 Forces and torques . . . . . . . . . . . . . . . . . . . . . . . . 7

3 Configurations 9

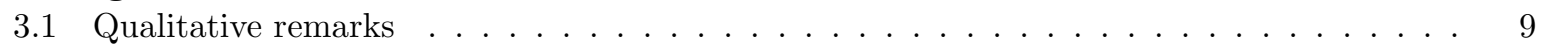

$3.2 \mu<\mu_{0} \ldots \ldots \ldots \ldots \ldots \ldots \ldots$

$3.3 \quad \boldsymbol{\mu} \geq \mu_{0} \ldots \ldots \ldots \ldots \ldots \ldots \ldots \ldots$

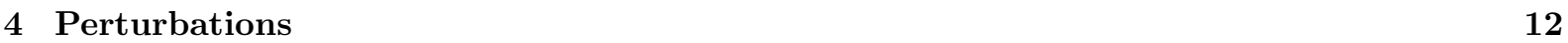

4.1 Equilibrium-connecting deformations . . . . . . . . . . . . . . . . . . . . . . . . . . . . . . 12

4.2 The $\sigma$-identities $\quad \ldots \ldots \ldots \ldots \ldots \ldots$

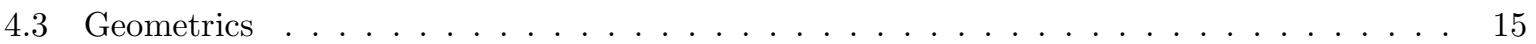

4.4 Self-intersections . . . . . . . . . . . . . . . . . . . . . . . . . . . . . . . 17

4.5 Connecting equilibria . . . . . . . . . . . . . . . . . . . . . . . . . . . . . . . 18

4.6 Perturbing the circle $\ldots \ldots \ldots \ldots \ldots \ldots$

5 Conclusions 21

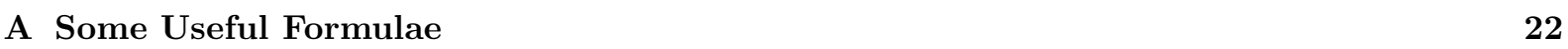

\begin{tabular}{|ll}
\hline B Averages & 23
\end{tabular}

\section{Introduction}

Our object of study in this paper is a deflated rigid loop (elastica hypoarealis). To explain this, consider a closed loop in a plane. The loop is made of some elastic material and, if taken thin enough, the contribution of its longitudinal deformations to its elastic energy can be ignored. This gives rise to a hamiltonian density proportional to the square of its curvature - hence elastica. It is clear that fixing only the length $L$ of the loop will not lead to any surprises, the only possible equilibrium being a circle. What will happen though if we, in addition, fix its area $A$ to be less than that of the circle? (hence hypoarealis).

This particular hamiltonian (without the constraints) has a distinguished history, dating back at least to the Bernoullis. It makes a reappearance in Euler's inspired analysis of a mundane but subtle problem in mechanical engineering: the buckling of a loaded beam 25, 9. More recently, variants of this problem have attracted the attention of mathematicians. In particular, the problem of determining the curves of constant length which minimize the bending energy, on surfaces of constant curvature, is considered in Refs. 10, 4, 15, while interconnections with knot theory are explored in [12]. Adding the constraint on the enclosed area, as we propose here, provides a particularly fruitful generalization of this work. 
Hamiltonians depending on extrinsic curvature have also been studied in statistical physics [2, 21. For space curves, an effective hamiltonian which depends on twist as well as bending provides a phenomenological description of stiff polymers, and in particular DNA (see e.g. Refs. 117, 3]). Moving up one dimension, the leading term in the Helfrich hamiltonian, which describes the equilibrium configurations of lipid membranes, is proportional to the square of the traced extrinsic curvature $K$ integrated over the surface, namely, the conformally invariant Willmore functional [26, 11, 20, 22]. In the variant of the Helfrich model proposed by Svetina and Zeks, the so-called bilayer couple model, constraints are placed on the surface, such as constant area, constant enclosed volume, and constant integrated mean curvature (the latter breaking the $K \rightarrow-K$ symmetry of the problem) [23.2] However, the equations which determine the equilibrium are highly non-trivial higher-order PDEs. One motivation for introducing our variant of the elastica, as a planar analogue of the above, is its potential as a toy model for understanding these higher dimensional membranes. At the simplest level, if a closed membrane possesses a symmetry along the $z$-axis, its profile at any $z$ will be a fixed loop. The dimensionally reduced Helfrich hamiltonian is then the bending energy of this loop. It is possible to examine the loop hamiltonian exactly. While a lot is known about axially symmetrical closed configurations [6], there is very little non-perturbative knowledge of the equilibria which exist when axial symmetry is broken: the loop configurations provide an analytic point of entry.

An additional application of the planar problem was pointed out recently by Willmore: any closed loop can be exploited to generate an axially symmetric toroidal geometry. The equilibria of the loop hamiltonian on a surface of constant negative curvature can be mapped into equilibria of the Helfrich functional 27].

The feature of our model we will exploit is its integrability, very well disguised when the problem is cast with respect to the variables embedding the loop in the plane. Indeed, the determination of the curvature at equilibrium can be reduced to the study of the motion of a particle in a one-dimensional quartic potential. The curvature can be solved as a quadrature in terms of elliptic integrals. Remarkably, we discover that the problem possesses a second, far less-obvious, level of integrability: the embedding can be expressed as a local function of the curvature without any need of the two integrations one would have expected. As a consequence, the equilibrium configuration is given by a geometrical construction of pythagorean simplicity.

The condition of closure of the loop results in a discrete spectrum for a given constrained perimeter and area. We show that scaling can be exploited to fix one of these, the perimeter say. One branch of the spectrum then consists of configurations with an $n$-fold symmetry $(n=2,3, \ldots)$ which inflate into a circle as the enclosed area is increased. If the area is evacuated these configurations will eventually self-intersect, tending to a limiting configuration of an inverted (i.e., negative area) circle, winding $n-1$ times and decorated with $n$ infinitesimally small circles. The latter dominate, in this limit, the energy and give rise to a pole in the energy versus area diagram, the residue of which we evaluate exactly for each $n$.

The remaining branches of the spectrum correspond to rather complicated self-intersecting pairs which bifurcate from a limiting configuration. While self-intersecting configurations are undoubtedly of interest mathematically, they are not of primary relevance to the physical problem we pose - we determine sufficient conditions for avoiding them (nevertheless, we describe a physical situation where they may arise).

In general, one would expect equilibria connecting perturbations to be governed by a fourth order differential equation. We show, however, that this equation can be reduced (by three orders) to a first order one. The latter is singular at the circular configuration, where bifurcation occurs. Analytical approximations for the energy as a function of the area are derived, in the limit of sinusoidal perturbations of the circle of frequency $n$. Combined with the pole mentioned above, these expressions provide a reliable sketch of the energy - area relationship for each $n$.

\footnotetext{
${ }^{2}$ We note that there is no genuine one-dimensional analog of its refinement, the so-called area difference model $[22$.
} 
The paper is organized as follows: Sect. 2 introduces the model and the shape equation that determines equilibria. We show how it can be reduced to the motion of a fictitious classical particle in a quartic potential. We address the behaviour under scaling of the shape equation and we show that the problem has essentially one free parameter. Moreover we analyze the loop statics; this provides a physical interpretation for the shape equation itself. In Sect. 3 we study the configuration space for this model. In particular, we describe the angle $\Theta_{0}$ by which the normal to the loop gets rotated in a full oscillation of the fictitious particle in the potential. Equilibria connecting perturbations is the subject of Sect. 4 . These are determined by a complicated fourth order differential equation, which is used to obtain the purely geometrical construction mentioned above. In turn, the latter permits the reduction of the order of the original equation from four to one, as well as the derivation of a sufficient condition for non-self-intersections. Finally, we obtain analytical expressions for the energy as a function of the area, in some limiting regimes, which allow for a reliable sketch of its behaviour. In the appendices we collect various expressions, useful in the calculation of variations, we derive a recursion relation for the average of the powers of $K$ and comment on an interesting Legendre transform in the space of parameters of the model.

\section{First Considerations}

\subsection{Energy functional}

A closed loop, parametrized by $s \in[0,1]$, is described by the embedding in the plane,

$$
\vec{x}=\vec{X}(s) .
$$

The arc length $\ell$ along the loop is given by

$$
\ell(s)=\int_{0}^{s} d s^{\prime}\left(\frac{d \vec{X}}{d s^{\prime}} \cdot \frac{d \vec{X}}{d s^{\prime}}\right)^{1 / 2} .
$$

The most general expression for the energy of the configuration $X(s)$ which $i$ ) does not depend on the parametrization, $i$ i) involves no higher than two derivatives of $\vec{X}$ and $i i i)$ is quadratic in these derivatives, is given by

$$
F[\vec{X}]=\alpha \int d \ell K^{2},
$$

where $K$ is the geodesic curvature, equal to the inverse of the radius of curvature at each point of the loop. We will take henceforth $\alpha$ to be equal to $1-\alpha$ is dimensionful, unlike its two-dimensional analogue, and this means we are measuring length in units of $\alpha$. Let $\hat{t}$ be the unit tangent to the loop (transversed counterclockwise) and $\hat{n}$ its outwards normal. Then we have the Frenet-Serret equations for a plane curve (the prime denotes a derivative with respect to arc length $\ell$ ),

$$
\hat{n}^{\prime}=K \hat{t}, \quad \hat{t}^{\prime}=-K \hat{n} .
$$

In terms of the angle $\Theta$ that $\hat{n}$ makes with, say, the $x$-axis, $K=\Theta^{\prime}$.

To implement the constraints of fixed length and enclosed area, we introduce the constrained functional:

$$
F_{c}[X]=F[X]+\mu\left(\int d \ell-L\right)+\sigma\left(\int_{\text {int }} d^{2} x-A\right) .
$$

\footnotetext{
${ }^{3}$ Notice that the opposite sign convention for $K$ is quite common in the literature.
} 
$\mu$ appears as a lagrange multiplier enforcing the constraint fixing the length of the loop to some value, $L$. In the same way, $-\sigma$ is associated with the constraint fixing the enclosed area to the value $A$. We note that, in general,

$$
\mu=-\frac{\partial F_{c}(L, A)}{\partial L}, \quad \sigma=-\frac{\partial F_{c}(L, A)}{\partial A} .
$$

In particular, if the area constraint is relaxed so that $\sigma=0$, then $\partial F_{c}(L, A) / \partial A=0$. Looking at (5), one might be led to identify $\mu, \sigma$ with the tension and differential pressure on the loop but this is only half true - we discuss the physical meaning of these parameters in Sec. 2.5. We have not included, in our energy functional, a term proportional to the integral of $K$. This is because such a term is simply the total angle by which $\hat{n}$ gets rotated in transversing the loop once, equal to $2 \pi$ for a non-self-intersecting loop.

We emphasize that the bending energy is not scale invariant (notice that neither is the dimensionally reduced Helfrich hamiltonian). An unconstrained circular loop will expand without limit. The natural scale invariant expression, $\int d \ell|K|$, though not in itself a topological invariant, does have a vanishing Euler-Lagrange derivative almost everywhere. Taken as an energy functional, its constrained configurations will be arcs of a circle (solutions of $\mu K=\sigma$ ) joined together at curvature discontinuities in such a way as to mimimize the corresponding constrained energy function. This model will be considered elsewhere.

\subsection{Shape equation}

The Euler-Lagrange equations follow from extremizing $F_{c}[X]$,

$$
\frac{\delta F_{c}}{\delta X^{\mu}}=0 \text {. }
$$

The reparametrization invariance of $F_{c}[X]$ implies that the tangential projection of these equations is vacuous. The normal projection gives

$$
2 K^{\prime \prime}+K^{3}-\mu K-\sigma=0
$$

(see the appendix for some relevant formulae). We note that with the identification, $\mu \rightarrow \mu+K_{0}^{2}$, Eq. (7) coincides with Eq. (7) of [18] (see also [19]). Is is worth pointing out that a loop on a surface of constant Gaussian curvature $G$ satisfies the above equation with $\mu \rightarrow \mu-2 G$.

A plane curve is determined, up to rigid motions, by its curvature [7]. As is well known, our onedimensional system is completely integrable. This is obvious because we have cast derivatives with respect to arclength. With respect to an arbitrary parametrization of the loop, first derivative terms associated with the one-dimensional Laplacian appear which conceal this fact. Writing (7) in the form

$$
K^{\prime \prime}=-\frac{d}{d K} V(K), \quad V(K) \equiv \frac{1}{8} K^{4}-\frac{\mu}{4} K^{2}-\frac{\sigma}{2} K,
$$

we map our problem onto the problem of determining the motion of a fictitious particle in a quartic potential, $K$ being the displacement of the particle and $\ell$ playing the role of time. The total energy $E$ of the particle is conserved,

$$
\frac{d E}{d \ell}=0, \quad E \equiv \frac{1}{2} K^{\prime 2}+V(K),
$$

a fact that permits the expression of $K^{\prime}$ in terms of $K$ and hence, of the arclength along the loop as an integral over $K\left(\right.$ via $\left.d \ell=d K / K^{\prime}\right)$

$$
\ell=\int \frac{d K}{\sqrt{2(E-V(K))}}
$$


We emphasize the difference between the energy $E$ of the fictitious particle, on the one hand, and the bending energy $F$ of the loop, on the other. In particular, the configuration of least 'energy' in the analogue is not the minimum of the hamiltonian.

The motion of the particle in the potential $V$ is periodic. Closed loop configurations consist of an integer number of identical segments, each one corresponding to one full oscillation of the particle, and hence made up itself of two symmetric halves. Then, the condition of closure of the loop can be expressed as

$$
\Theta_{0}=\frac{2 \pi}{n}, \quad n=2,3, \ldots
$$

where $\Theta_{0}$ is the angle by which the normal $\hat{n}$ gets rotated in one full oscillation of the particle, given by

$$
\Theta_{0}=2 \int_{K_{\min }}^{K_{\max }} \frac{K d K}{\sqrt{2(E-V(K))}}
$$

( $K_{\min }, K_{\max }$ denote the turning points). The equilibrium configurations have $n$-fold symmetry and a well-defined center. The value $n=1$ is omitted in (11) because it is special - see below. We examine in detail the resulting configurations in Sect. 3 .

\subsection{Scaling}

Let us examine the behavior of Eq. (7) under scaling of the position vector, $\vec{X} \rightarrow \lambda \vec{X}$. We find

$$
\ell \rightarrow \lambda \ell, \quad \frac{d}{d \ell} \rightarrow \frac{1}{\lambda} \frac{d}{d \ell}, \quad K \rightarrow \frac{1}{\lambda} K .
$$

Let $\vec{X}(\ell)$ correspond to some given solution of Eq. (7), with parameter values $\left(\sigma_{1}, \mu_{1}\right)$. Then, a scaled solution with the same shape, $\lambda \vec{X}(\lambda \ell)$ and with $\left(L_{1}, A_{1}, F_{1}, E_{1}\right) \rightarrow\left(\lambda L_{1}, \lambda^{2} A_{1}, \lambda^{-1} F_{1}, \lambda^{-4} E_{1}\right)$, is obtained by rescaling the multipliers as follows:

$$
\mu_{1} \rightarrow \frac{1}{\lambda^{2}} \mu_{1} \equiv \mu_{\lambda}, \quad \sigma_{1} \rightarrow \frac{1}{\lambda^{3}} \sigma_{1} \equiv \sigma_{\lambda} .
$$

Eliminating $\lambda$ we find the orbits of scaling in the $(\sigma, \mu)$-plane

$$
\mu_{\lambda}=\frac{\mu_{1}}{\sigma_{1}^{2 / 3}} \sigma_{\lambda}^{2 / 3}
$$

Furthermore, an inversion in the origin, $X^{\mu} \rightarrow-X^{\mu}$ can be identified with a rescaling by $\lambda=-1$, which maps a solution with a given $\sigma$ into one with $-\sigma$. It follows that, for the purpose of identifying distinct configurations at least, one can set, e.g. $\sigma=1$ and scan the essentially one-dimensional configuration space by varying $\mu$. For each value of $\mu$, which fixes the form of the potential $V(K)$, one has still to vary the energy $E$ of the particle in the well so as to satisfy (11), resulting, in general, in an infinite discrete spectrum "above" the point $(1, \mu)$ in the $(\sigma, \mu)$-plane.

\subsection{Windings}

The closed configurations we wish to classify possess two topological invariants, both given as the winding numbers of maps $S^{1} \rightarrow S^{1}$. The first such map is the normal map of the loop, associating with each value of the parameter $s$ (which ranges over the first $S^{1}$ above) the corresponding value of $\hat{n}$ (which ranges over the unit circle, the second $S^{1}$ above). For each rotation of $s, \hat{n}$ will generally rotate $m$ times, with $m$ an integer. Configurations with distinct $m$ 's are topologically isolated, the physical implementation 

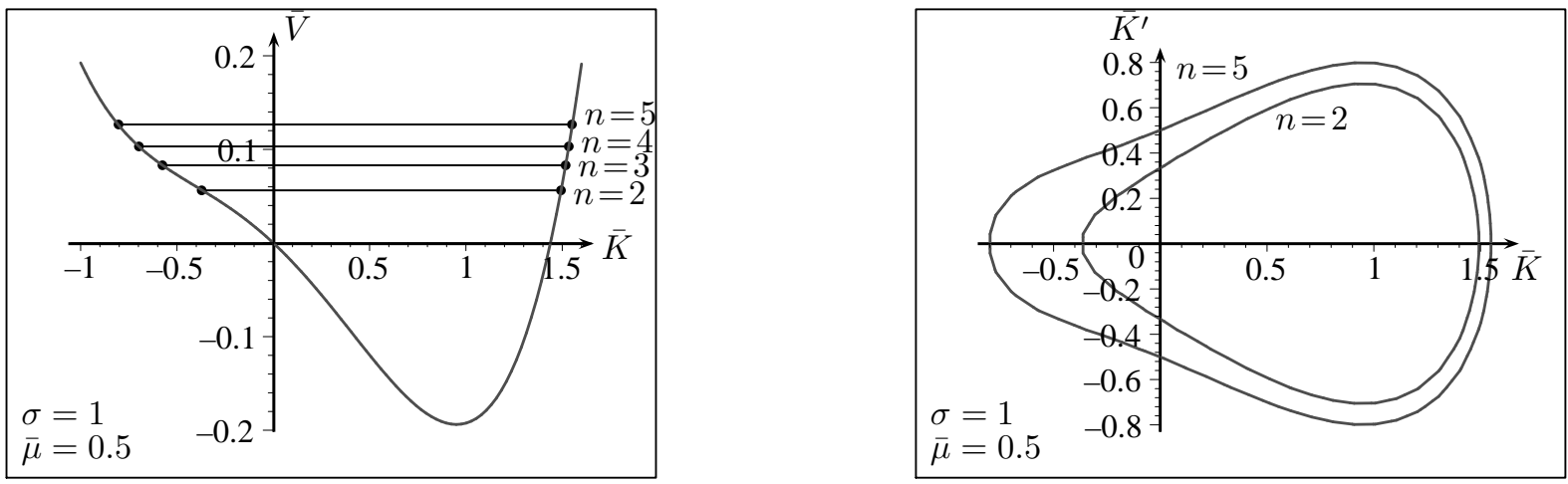

Figure 1: On the left: the potential $\bar{V}(\bar{K})$, for the values of $\bar{\mu}, \sigma$ shown. Barred quantities, in this and subsequent figures, are measured in units of $\mu_{0}^{-q / 2}, q$ being their length dimension. Also shown, with horizontal lines, are the energies $\bar{E}^{(n)}$ that give rise to the $n=2,3,4,5$ closed configurations, equal to $0.253,0.278$, $0.298,0.320$ respectively (in units of $\mu_{0}^{2}$ and measured from the bottom of the well - compare with the $\bar{\mu}=0.5$ curve in Fig. (4). On the right: the closed trajectories in the phase plane $\bar{K}-\bar{K}^{\prime}$ of the ficticious particle, corresponding to oscillations in the potential $V$ with energies $\bar{E}^{(2)}, \bar{E}^{(5)}$ - the $n=3,4$ trajectories lie in between the two shown.

being the infinite energy barrier associated with the move $\Omega \rightarrow \longrightarrow$ Nonself-intersecting loops correspond to normal maps with index $m= \pm 1$ (the converse is not true).

The second map is from the $s$-circle to the closed orbit $\left(\sim S^{1}\right)$ traced by the phase point in the plane $\left(K, K^{\prime}\right)$. For a configuration corresponding to $n$ complete oscillations of the particle, the phase point goes around the closed curve $\frac{1}{2} K^{\prime 2}+V(K)=E n$ times, Fig. 1. There is a finite energy barrier separating configurations with distinct $n$ 's which prevents transitions between them.

\subsection{Forces and torques}

What can we learn about the loop configurations by looking at the equilibrium of forces and torques on an infinitesimal loop segment? First we ask, why would a rigid loop want to have area less than the maximal allowed by its length? One setup that supplies an answer is to imagine that our loop is actually the cross-section of an infinite cylinder, the interior and exterior of which are filled with an incompressible fluid. We start by filling the cylinder to its maximum capacity, this gives a circular loop. We then take out some fluid from the interior while making sure that the external pressure is large enough so as to prevent the formation of bubbles in the interior. Notice that, because of its rigidity, the cylinder takes up some part of the exterior pressure and only transmits to its interior a fraction of it, it's the differential pressure that crumbles the cylinder walls. The second of (5) then points to the identification of $-\sigma$ with this differential pressure, taking into account that the latter points inwards. One has to be careful in applying the same argument to $\mu$. The first of (可) seems to suggest that $\mu$ is the tension of the loop but this would presuppose the possibility of tangential deformations - there is nothing in our energy functional that tells us how much energy these cost and, indeed, we have already assumed that the loop cannot be stretched or compressed tangentially. The derivative in the first of (5) is computed by comparing distinct loops with infinitesimally differing lengths, it does not refer to the deformation of a single loop. With this in mind, we now turn to loop statics.

We denote by $\vec{T}\left(\ell_{0}\right)$ the total force from the segment of the loop with $\ell<\ell_{0}$ to the one with $\ell>\ell_{0}$ and by $\tau\left(\ell_{0}\right)$ the corresponding torque. The latter is equal to $-2 K$ - this follows from our normalization 

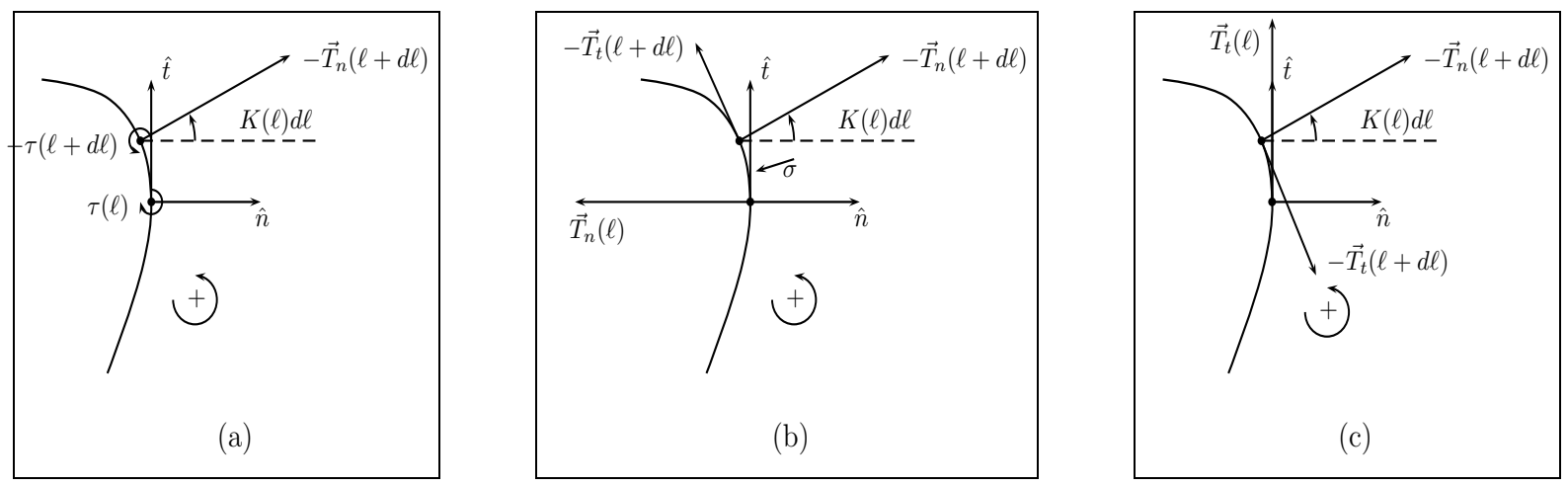

Figure 2: Balancing of (a) torques, (b) normal forces and (c) tangential forces on a segment of the loop. $K$ and $K^{\prime}$ are sketched positive, the resulting directions of torques and forces are shown. For $T_{t}$ this data is not enough, we have assumed additionally $K^{2}>\mu$ - this puts the center of the loop somewhere towards the left of each picture.

of the bending energy $F$. Then, balancing the torques on a segment extending from $\ell$ to $\ell+d \ell$ we get

$$
\tau(\ell)-\tau(\ell+d \ell)+T_{n}(\ell+d \ell) d \ell=0 \quad \Rightarrow \quad \tau^{\prime}-T_{n}=0,
$$

where $T_{n}$ is the normal force, the reference point was taken at $\ell$ and the sign conventions are shown in Fig. 2. The equilibrium of normal forces on the segment gives

$$
T_{n}(\ell)-T_{n}(\ell+d \ell)+T_{t}(\ell+d \ell) K(\ell) d \ell-\sigma d \ell=0 \quad \Rightarrow \quad-T_{n}^{\prime}+K T_{t}-\sigma=0,
$$

while the tangential components give

$$
T_{t}(\ell)-T_{t}(\ell+d \ell)-T_{n}(\ell+d \ell) K(\ell) d \ell=0 \quad \Rightarrow \quad T_{t}^{\prime}+K T_{n}=0 .
$$

Notice how, in (17) and (18), the curvature $K$ is responsible for the tangential force at $\ell+d \ell$ contributing a normal component at $\ell$ and vice-versa. Solving the above system of equations we find

$$
\tau=-2 K, \quad T_{n}=-2 K^{\prime}, \quad T_{t}=K^{2}-\mu
$$

where, in the third relation, the integration constant was fixed to the value $\mu$ by the requirement that one recover the differential equation for $K$, Eq. (7). We see from (19) that $-\mu$ is the tension of the loop at its inflection points, if any. Notice also how (7) is obtained upon substitution of the last two of (19) in (17), thereby identifying the physical origin of each of the terms in the former. In a forthcoming publication, where we extend our considerations to the case of a loop in space, it is shown how the above expressions for the forces and torque follow, in a model independent way, from an application of Noether's theorem.

Our submerged cylinder model for the loop leaves no room for self-intersecting configurations, could there be any use for these? Imagine the loop made of superconducting material, in the presence of a uniform magnetic field perpendicular to its plane. In the limit where the magnetic flux due to selfinductance is negligible compared to the one due to the external field, the area of the loop has to be constant to keep the flux constant. One can then adjust the area by changing the magnetic field and, for small enough areas, self-intersecting configurations will appear. 


\section{Configurations}

\subsection{Qualitative remarks}

For the purposes of this section, we may set, as explained above, $\sigma=1$ in the expression for the potential $V(K)$. Its critical points are given by the zeros of its derivative, i.e., by the roots $K_{i}, i=1,2,3$, of

$$
K^{3}-\mu K-1=0 .
$$

We find

$$
K_{1}=-K_{+}+i \sqrt{3} K_{-}, \quad K_{2}=-K_{+}-i \sqrt{3} K_{-}, \quad K_{3}=2 K_{+},
$$

where

$$
K_{+} \equiv \frac{m}{12}+\frac{\mu}{m}, \quad K_{-} \equiv \frac{m}{12}-\frac{\mu}{m}, \quad m^{3} \equiv 108+108 \sqrt{1-\frac{\mu^{3}}{\mu_{0}^{3}}},
$$

where $\mu_{0} \equiv 3 / 2^{2 / 3}$. Of these, $K_{3}$ is always real while the first two are real only for $\mu \geq \mu_{0}$. Notice that, for any $\mu$, the effect of the quadratic and the linear term in the potential is relatively important only in a neighborhood of the origin. For motions of the particle with sufficiently high energy $E$, the time spent by the particle in this region is negligible and the quartic term dominates the motion. As a result, we may conclude that $\Theta_{0}$ approaches zero with increasing $E$, due to the approximate symmetry $K \rightarrow-K$ of the motion. The term linear in $K$ spoils this symmetry and, for $\sigma>0$ (as taken in this section), makes $\Theta_{0}$ slightly positive for large $E$. As $\Theta_{0}$ descends, with increasing $E$, from this positive value to 0 , it will cross all critical values $2 \pi / n$ for $n$ greater than some $n_{0}$. We expect therefore to encounter configurations with arbitrarily high $n$, for all $\mu$ - see Fig. 4 .

On the other extreme, when the energy $E$ is only slightly greater than a local minimum of the potential (at $K_{1}$ or $K_{3}$ ), and the particle oscillates around that minimum, we may approximate $V(K)$ by a quadratic expression in $K$ and find for $\Theta_{0}$ the limiting value

$$
\Theta_{0 i}^{(\max )}=2 \pi \frac{\sqrt{2}}{\sqrt{3-\frac{\mu}{K_{i}^{2}}}}, \quad i=1,3
$$

where the superscript (max) is used because, as we shall see shortly, this is actually a maximum of $\Theta_{0 i}(\mu, E)$, for fixed $\mu$. Starting from (21), one infers the limiting values

$$
\Theta_{01}^{(\max )}\left(\mu \rightarrow \mu_{0}^{+}\right)=-\infty, \quad \Theta_{01}^{(\max )}(\mu \rightarrow \infty)=-2 \pi
$$

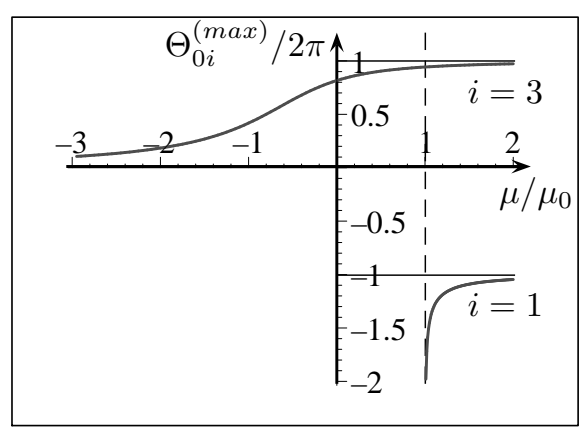

as well as

$$
\Theta_{03}^{(\max )}(\mu \rightarrow-\infty)=0, \quad \Theta_{03}^{(\max )}(\mu=0)=2 \pi \sqrt{\frac{2}{3}}, \quad \Theta_{03}^{(\max )}(\mu \rightarrow \infty)=2 \pi .
$$

A plot of $\Theta_{0 i}^{(\max )}(\mu)$ is given in Fig. 3. We notice that, for $\mu>\mu_{0}$, there are no non-self-intersecting configurations corresponding to oscillations in the left well.

We explain now why the value $n=1$ was omitted in (11). The reasoning behind that relation was that a closed configuration should correspond to $n$ complete oscillations of the particle. This is not necessarily so in the case of the circle. Any number of "oscillations" (of zero amplitude) will fit into a circle, including irrational numbers, and indeed, (23) shows that the circle corresponding to the particle resting at $K_{i}$, $i=1,3$, is made of $\sqrt{3-\frac{\mu}{K_{i}{ }^{2}}} / \sqrt{2}$ complete oscillations.

To examine what happens for values of $E$ that render the quadratic and linear terms important, we analyze the cases $\mu<\mu_{0}$ and $\mu \geq \mu_{0}$ separately. 


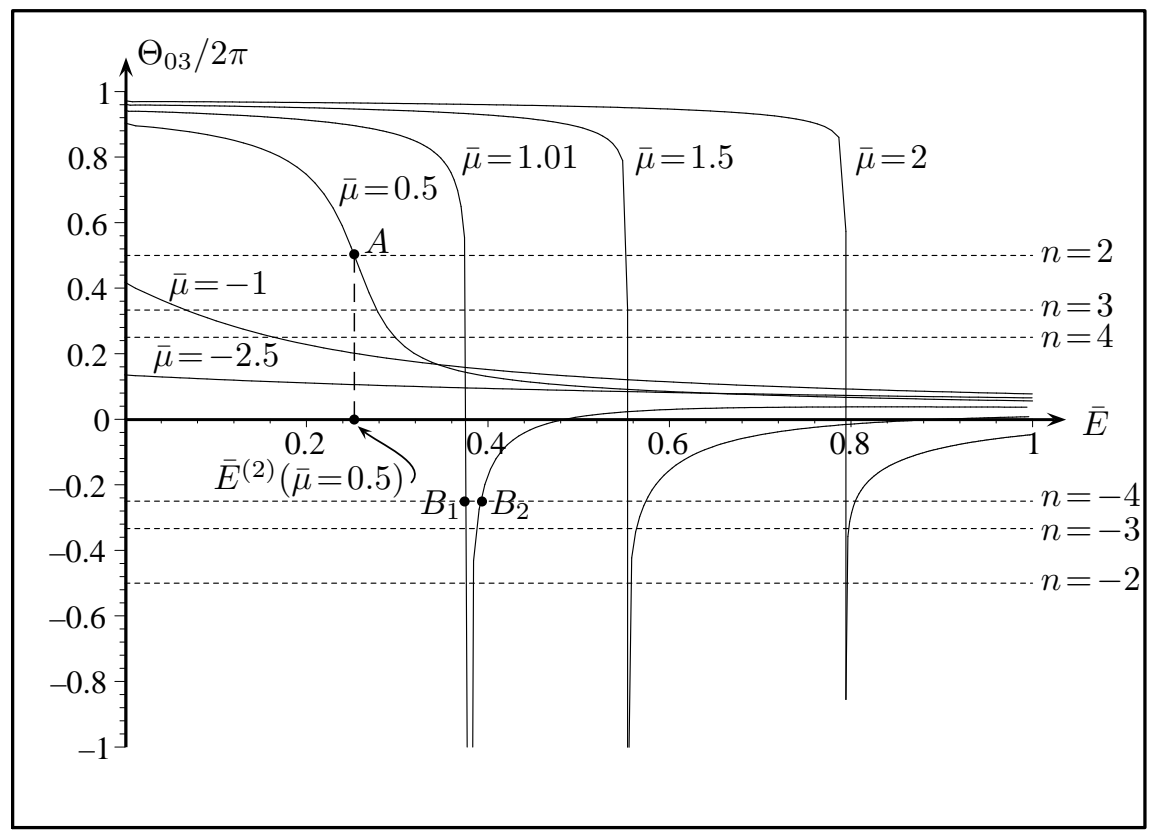

Figure $4: \Theta_{03}(\bar{E}), \bar{E} \equiv\left(E-V\left(K_{3}\right)\right) / \mu_{0}^{2}$, for various values of $\bar{\mu} \equiv \mu / \mu_{0}$. Also shown, with horizontal dashed lines, are some of the values of $\Theta_{03}$ that give rise to closed configurations. The corresponding values of $\bar{E}$ can be read off as in the case $\bar{\mu}=0.5, n=2$ shown - the (self-intersecting) configuration itself appears in Fig. 9 . Notice how the $\bar{\mu}=-1,-2.5$ curves miss the $n=2, n=2,3,4$ configurations respectively.

\section{$3.2 \mu<\mu_{0}$}

The potential possesses only one minimum, at $K_{3}>0$ given by (21) (see Fig. 田). $\Theta_{03}$ starts at $\Theta_{03}^{(\max )}$, for $E=V\left(K_{3}\right)$, and for $\mu$ far from $\mu_{0}$, decreases monotonically to zero with increasing $E$. The configurations that do not appear are those for which $2 \leq n<2 \pi / \Theta_{0}^{(\max )}$ as well as all with negative $n$. This condition determines a set $\left\{\mu_{n} \mid n=2,3, \ldots\right\}$ of critical values of $\mu$, such that, for $\mu<\mu_{n}$, all configurations with $n^{\prime}=2,3, \ldots, n$ are absent (the circle is, of course, always there). As $\mu$ approaches $\mu_{0}$, the left wall of the potential develops a plateau that tends to the horizontal, as $\mu \rightarrow \mu_{0}$. The particle spends a relatively long time in this (negative $K$ ) region, which results in a negative bump in the $\Theta_{0}$ curve. As $\mu \rightarrow \mu_{0}$, the minimum of this bump tends to $-\infty$ - negative $n$ configurations appear accordingly. Direct evaluation of the integral (12) gives [5]

$$
\Theta_{03}(\mu, E)=\frac{8 g(a B+b A)}{A-B}\left[\alpha_{2} \mathcal{K}(k)+\frac{\alpha-\alpha_{2}}{1-\alpha^{2}} \Pi\left(\frac{\alpha^{2}}{\alpha^{2}-1}, k\right)\right],
$$

where $k^{2}=\frac{(a-b)^{2}-(A-B)^{2}}{4 A B}$,

$$
\begin{aligned}
& g=\frac{1}{\sqrt{A B}} \\
& \alpha=\frac{A-B}{A+B} \\
& \alpha_{2}=\frac{b A-a B}{a B+b A} \\
& A^{2}=\left(a-b_{1}\right)^{2}+a_{1}^{2} \\
& a_{1}^{2}=\frac{4}{a+b}+a b-\frac{1}{4}(a+b)^{2} \\
& B^{2}=\left(b-b_{1}\right)^{2}+a_{1}^{2} \\
& b_{1}=-\frac{1}{2}(a+b) \\
& a=K_{\max }(\mu, E) \\
& b=K_{\min }(\mu, E)
\end{aligned}
$$


and $\mathcal{K}, \Pi$ are the complete elliptic integrals of the first and third kind respectively, given by

$$
\mathcal{K}(k)=\int_{0}^{\pi / 2} \frac{d \theta}{\sqrt{1-k^{2} \sin ^{2} \theta}}, \quad \Pi\left(\alpha^{2}, k\right)=\int_{0}^{\pi / 2} \frac{d \theta}{\left(1-\alpha^{2} \sin ^{2} \theta\right) \sqrt{1-k^{2} \sin ^{2} \theta}} .
$$

The result (26) holds for $\mu>\mu_{0}$ as well, with the appropriate choice of $K_{\min }, K_{\max }$. A plot of $\Theta_{03}(\mu, E)$, for various values of $\mu$, appears in Fig. 1 .

A couple of remarks are in order at this point. Consider the point $A$ in Fig. 4 , which is the intersection of the $\bar{\mu}=0.5$ curve with the $n=2$ line, corresponding to an $n=2$ configuration. Imagine now that $\bar{\mu}$ is diminished continuously - the corresponding curve will move more or less downwards forcing $A$ to move to the left. The corresponding $\bar{E}$ then diminishes which means that the fictitious particle oscillates in the potential well with smaller amplitude. When $A$ hits the $\Theta_{03}$-axis, $\bar{E}$ is zero, the particle sits at the bottom of the well and the corresponding configuration becomes a circle. In other words, all configurations like the one corresponding to the point $A$ can be continuously deformed to a circle.

This is not the case though with the points $B_{1}, B_{2}$ in the same figure. Consider $B_{1}$ - it corresponds to an $n=-4$ configuration for $\bar{\mu}=1.01$. Imagine now that $\bar{\mu}$ is diminished in a continuous way. As soon as it becomes smaller then 1 , the infinite negative pole of the corresponding curve is softened to a negative minimum which, for $\bar{\mu}$ sufficiently close to 1 , still intersects the $n=-4$ line. Given that the $\mu$-curves in Fig. 1 become almost horizontal at high $E$, we give an exagerrated sketch of the situation in Fig. 司. As the value of $\bar{\mu}$ is lowered, this negative minimum rises and, for a critical value of $\bar{\mu}$, will just touch the $n=-4$ line, i.e., $B_{1}$ and $B_{2}$ collapse to a single point $B$. The configuration that corresponds to this point is not a circle, since $\bar{E}$ is positive and the particle oscillates with a finite amplitude, i.e., $K$ is not constant. We conclude that configurations corresponding to points like $B_{1}, B_{2}$ cannot be continuously deformed to a circle. Moreover, there exist bifurcation points, like $B$ above, distinct from the circle. When $\bar{\mu}$ is lowered even more, the minimum of

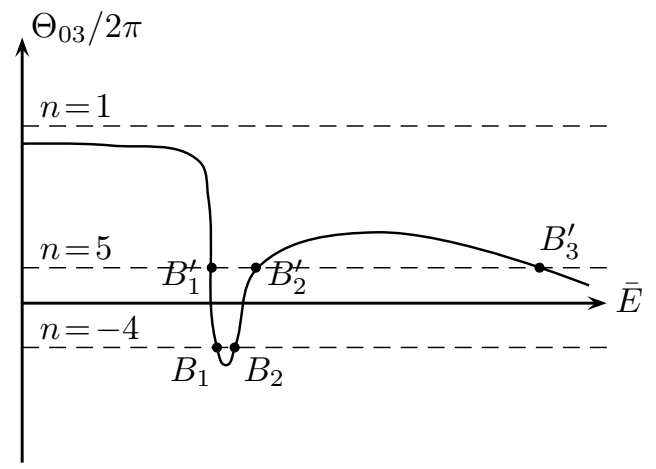

Figure 5: Bifurcations: as $\bar{\mu}$ is reduced, the minimum of the curve shifts upwards and $B_{1}, B_{2}$ collapse to a point. Further decrease of $\bar{\mu}$ forces $B_{1}^{\prime}$, $B_{2}^{\prime}$ to collapse to a point. The corresponding configurations cannot be continuously deformed to a circle.

the $\bar{\mu}$-curve becomes positive and remarks similar to the above can be made about its points of intersection $B_{1}^{\prime}, B_{2}^{\prime}$ with sufficiently high $n$ lines (see Fig. 可).

\section{3 $\mu \geq \mu_{0}$}

The potential posseses two local minima, at $K_{1}<0$ and $K_{3}>0$ (with $V\left(K_{1}\right)>V\left(K_{3}\right)$ ) and one local maximum at $K_{2}<0$ (see Fig. 6). Depending on its energy and where it is started from, the particle is confined in the left well, the right well or visits both during every oscillation.

We note the following, regarding the asymptotic behaviour of $\Theta_{0 i}$ as $\mu \rightarrow \infty$. In this regime, the linear term becomes negligible in $V(K)$ and the integral giving $\Theta_{0 i}$ can be easily seen to reduce (with $K^{2} \rightarrow x$ ) to an integral giving (half) the period of a harmonic oscillator in a potential $x^{2} / 4-\mu x / 2$, which is independent of its energy $E$, as well as the 'constant force' $\mu$. Another way to see the $\mu$-independence is by observing that, with $\sigma=0$ (which is equivalent to sending $\mu$ to infinity), one lies on the $\mu$-axis in the $(\sigma, \mu)$-plane, which is an orbit under scaling, hence changes in $\mu$ in this regime leave $\Theta_{0 i}$ invariant. Another novelty in this case is that, as the energy approaches $V\left(K_{2}\right)$ (either from above or below), the period of the motion tends to infinity, most of which the particle spends at $K_{2}$. Assume we start the 

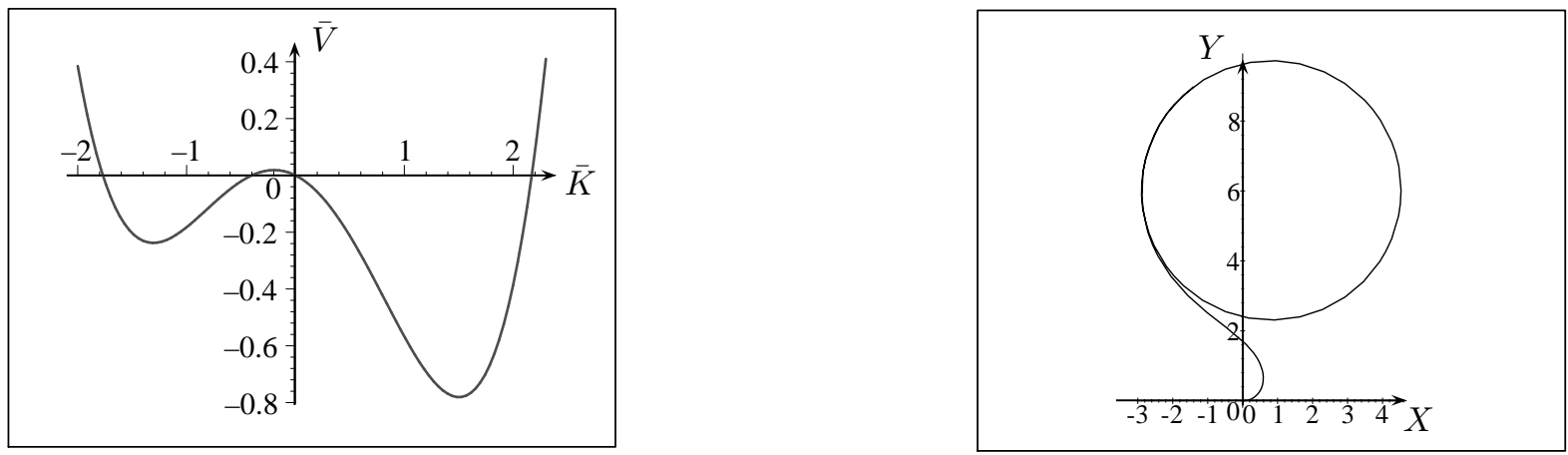

Figure 6: On the left: the potential $\bar{V}(\bar{K})$ for $\bar{\mu}=2$. On the right: spiralling configuration for $E=V\left(K_{2}\right)$, with the particle starting at the turning point on the right with zero velocity.

particle at the turning point on the right with zero velocity and with just the right energy to reach the central maximum of $V, E=V\left(K_{2}\right)$. The corresponding curve starts in a counterclockwise sense with decreasing curvature, passes through an inflection point and acquires infinite length spiralling forever clockwise as it approaches asymptotically a circle of radius $1 / K_{2}$, see Fig. 6 .

$\Theta_{03}$ tends accordingly to $-\infty$, this corresponds to the negative poles in Fig. 国. As mentioned already, for $V\left(K_{1}\right)<E<V\left(K_{2}\right)$, $\Theta_{0}$ will have two branches, $\Theta_{01}$ and $\Theta_{03}$, corresponding to the motions confined to either well. Both of these branches will tend to $-\infty$ as $E \rightarrow V\left(K_{2}\right)$. For $E>V\left(K_{2}\right)$ there is only one branch, which starts from $-\infty$ and approaches asymptotically zero (as $E \rightarrow \infty$ ), after having reached a positive local maximum. A three-dimensional plot of $\Theta_{03}(\mu, E)$ is given in Fig. 7. We present a representative collection of configurations in Fig. 8. We also show, for reference purposes, some self-intersecting configurations in Fig. 9 .

\section{Perturbations}

\subsection{Equilibrium-connecting deformations}

We study here the following problem: given an equilibrium configuration $\vec{X}$, find an infinitesimal deformation $\delta_{\epsilon} \vec{X}=\epsilon \hat{n}$ such that $\vec{X}+\delta_{\epsilon} \vec{X}$ describes a nearby equilibrium. Notice that $\delta_{\epsilon} \vec{X}$ is not necessarily the displacement vector of some point of the loop - the latter will generally have a tangential component as well. Our motivation is to obtain new solutions from known ones, with the closure condition automatically satisfied.

Using some of the formulae listed in the appendix, the linearization of the equilibrium condition (7) gives that the deformation $\epsilon$ must satisfy

$$
\begin{aligned}
D E_{4}(\epsilon) & \equiv 2 \epsilon^{\prime \prime \prime \prime}+\left(5 K^{2}-\mu\right) \epsilon^{\prime \prime}+10 K K^{\prime} \epsilon^{\prime}+\left(12 E-\frac{5}{2} K^{4}+6 \mu K^{2}+10 \sigma K\right) \epsilon \\
& =-\delta_{\epsilon} \mu K-\delta_{\epsilon} \sigma
\end{aligned}
$$

where the deformed configuration satisfies (7), with $\mu \rightarrow \mu+\delta_{\epsilon} \mu, \sigma \rightarrow \sigma+\delta_{\epsilon} \sigma$. The energy will change as well, $E \rightarrow E+\delta_{\epsilon} E$. This appears explicitly in the linearization of (\&), which gives

$$
\begin{aligned}
D E_{3}(\epsilon) & \equiv 2 K^{\prime} \epsilon^{\prime \prime \prime}+\left(K^{3}-\mu K-\sigma\right) \epsilon^{\prime \prime}+2 K^{2} K^{\prime} \epsilon^{\prime}+K\left(12 E-\frac{1}{2} K^{4}+2 \mu K^{2}+5 \sigma K\right) \epsilon \\
& =-\frac{1}{2} K^{2} \delta_{\epsilon} \mu-K \delta_{\epsilon} \sigma-\delta_{\epsilon} E .
\end{aligned}
$$




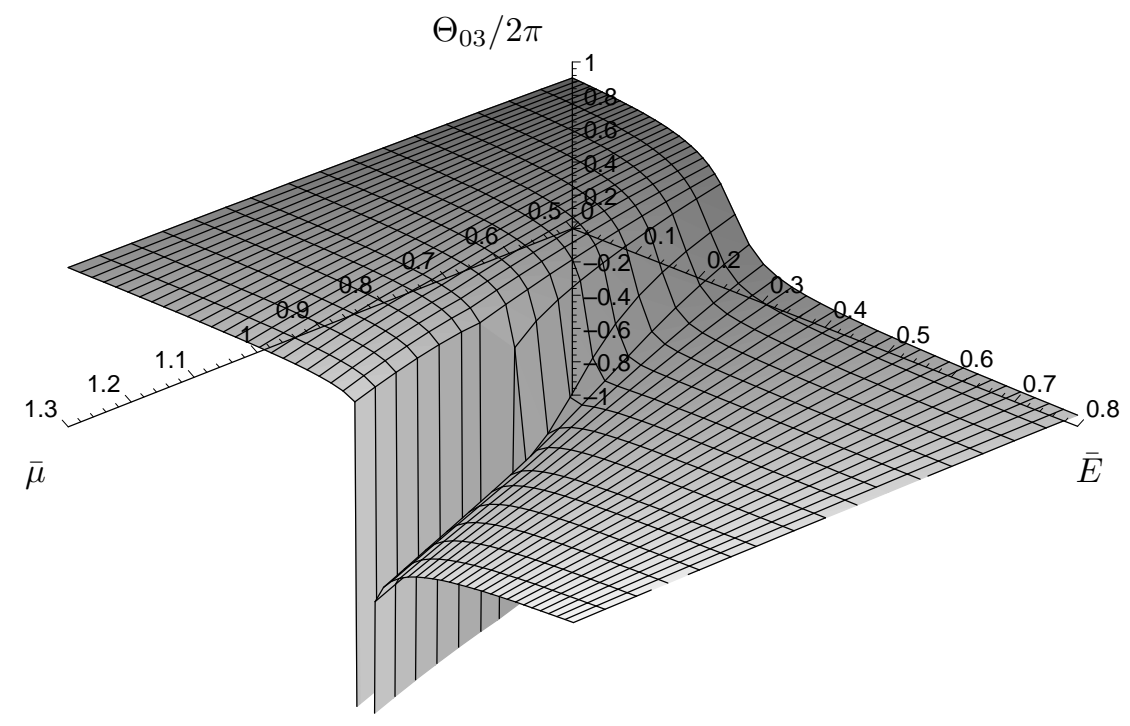

Figure 7: The angle $\Theta_{03}(\bar{\mu}, \bar{E})$, for $0.5 \leq \bar{\mu} \leq 1.3$, in steps of 0.05 and $0 \leq \bar{E} \leq .8$, in variable step. The $\bar{\mu}=1$ curve has been shifted to $\bar{\mu}=1.01$ in order to avoid the ambiguity in the number of roots of $\dot{V}$. The negative pole in the curves for $\bar{\mu}>1$ occurs at $E=V\left(K_{2}\right)$.

\subsection{The $\sigma$-identities}

We introduce a number of functions on the loop, relevant in the study of the solutions of (29). Any vector field $\vec{a}$ defines the following two functions on the loop

$$
a_{n} \equiv \vec{a} \cdot \hat{n}, \quad a_{t} \equiv \vec{a} \cdot \hat{t} .
$$

The position vector $\vec{X}$, in particular, supplies

$$
h \equiv \vec{X} \cdot \hat{n}, \quad p \equiv \vec{X} \cdot \hat{t},
$$

both functions evidently dependent on the choice of origin. One finds

$$
a_{n}^{\prime}=-K a_{t}, \quad a_{t}^{\prime}=K a_{n}, \quad h^{\prime}=K p, \quad p^{\prime}=1-K h,
$$

where $\vec{a}$ is henceforth assumed constant. We will say that a function $f$ on the loop is the generator of a transformation iff, under the latter, $\delta \vec{X} \cdot \hat{n}=f$. With this definition, $a_{n}, a_{t}$ are the generators of translations along $\vec{a}$ and perpendicularly to it respectively. $p$ and $h$ generate rotations and dilations respectively (both w.r.t. the origin). One easily verifies that $D E_{4}\left(a_{n}\right)=D E_{4}\left(a_{t}\right)=D E_{4}(p)=0$ while $D E_{4}(h)=2 \mu K+3 \sigma$, in accordance with the scaling behavior of $\mu, \sigma$ found earlier, Eq. (14). Experimenting a little with (29) we find, not without some surprise, two more solutions

$$
D E_{4}\left(K^{\prime}\right)=0, \quad D E_{4}\left(\sigma^{-1}\left(K^{2}-\mu\right)\right)=2 \mu K+3 \sigma .
$$

Noting, additionally, that when the origin is at the center of the loop, the zeros of $p$ coincide with the extrema of $K$, as well as the coincidence of the extrema of $h$ and $K^{2}$, we make the ansatz

$$
h=\sigma^{-1}\left(K^{2}-\mu\right)+f, \quad p=2 \sigma^{-1} K^{\prime}+g,
$$




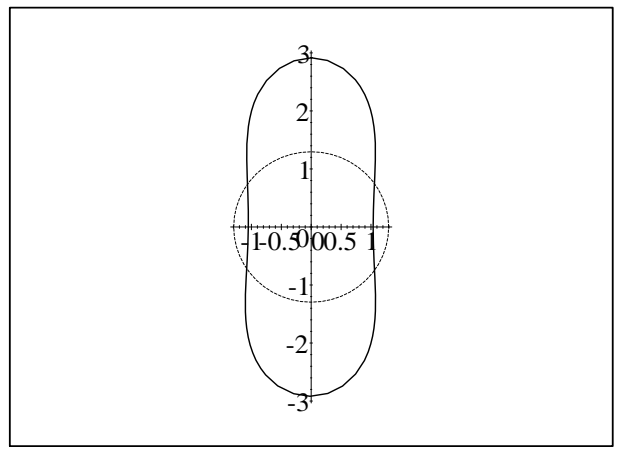

(a)

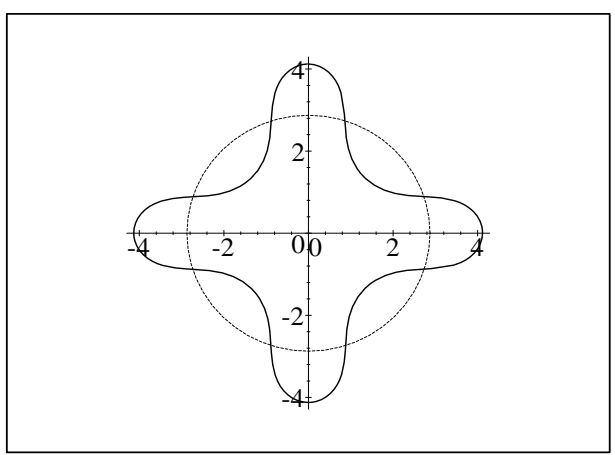

(c)

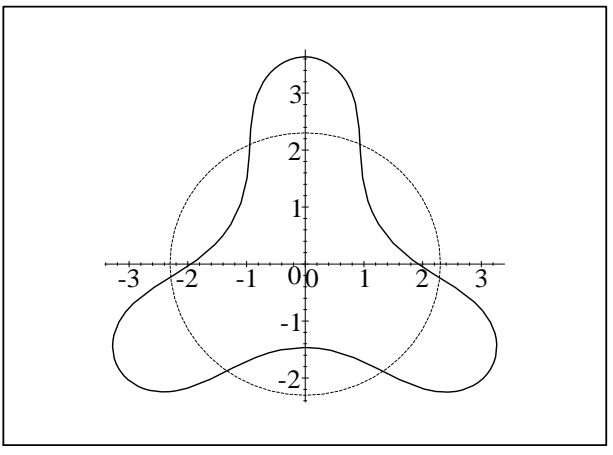

(b)

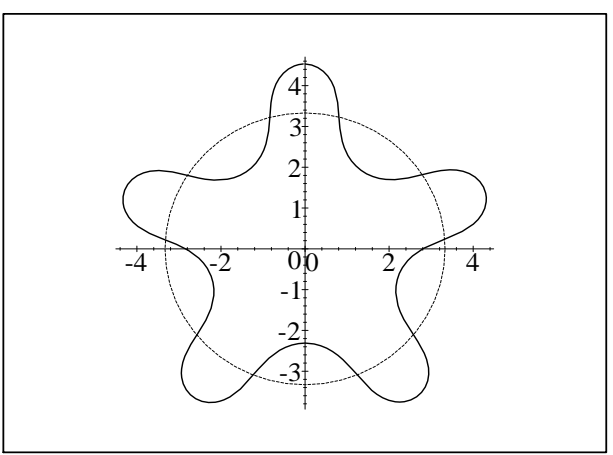

(d)

Figure 8: Non-self-intersecting configurations for $\bar{\mu}=-0.5, \sigma=1$ and $n=2,3,4,5$. Also shown, for each configuration, is the circle of radius $X_{0}$ (see Eq. (38)), passing through its inflection points.

with $f, g$ to be determined. Substituting in (33), we find $f^{\prime}=K g, g^{\prime}=-K f$, with solution

$$
f=a \cos \left(\Theta-\phi_{0}\right), \quad g=a \sin \left(\Theta-\phi_{0}\right),
$$

where $a, \phi_{0}$ are arbitrary constants. One recognizes $f, g$ to be, respectively, the functions $a_{n}, a_{t}$ corresponding to the position vector $\vec{a}$ of the center of the loop, then (35) state

$$
h=\sigma^{-1}\left(K^{2}-\mu\right)+a_{n}, \quad p=2 \sigma^{-1} K^{\prime}+a_{t},
$$

a remarkable geometrical property, the implications of which will occupy us shortly. Notice that neither of (37) survives in the $\sigma \rightarrow 0$ limit. Unless otherwise stated, we will take $\vec{a}$ equal to zero in what follows.

One might wonder whether (29) admits other polynomial solutions in $K$, apart from the second of (34). To investigate this, we rewrite (29) in terms of derivatives w.r.t. $K$, using $\frac{d}{d \ell}=K^{\prime} \frac{d}{d K}$ and find for $D E\left(K^{n}\right)$ the leading term $\left(\frac{1}{8} n^{4}+\frac{3}{4} n^{3}+\frac{1}{8} n^{2}-3 n-\frac{5}{2}\right) K^{n+4}$, with only positive integer root $n=2$. We conclude that no other polynomial solutions of (29) exist. 


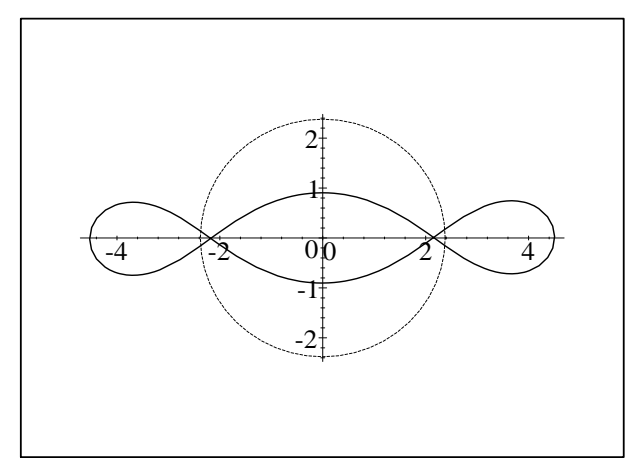

(a)

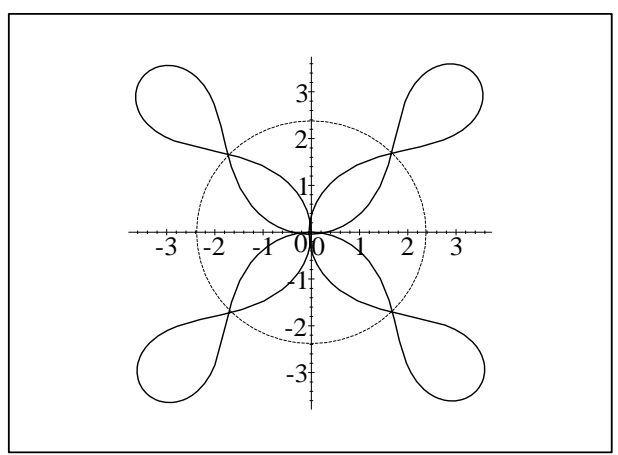

(c)

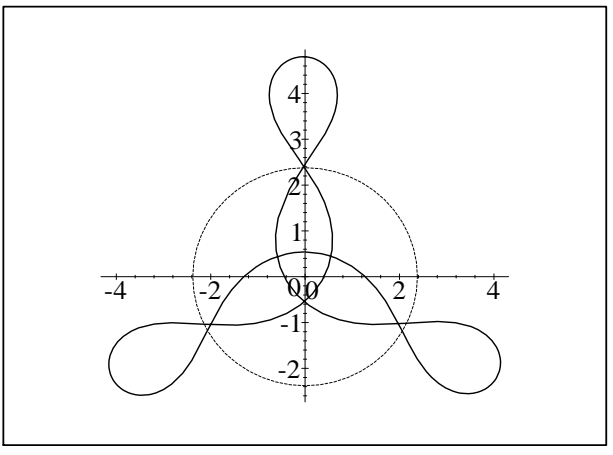

(b)

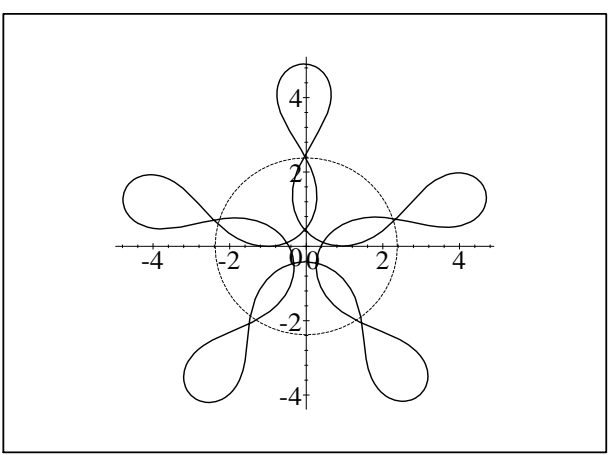

(d)

Figure 9: Self-intersecting configurations for $\bar{\mu}=0.5, \sigma=1$ and $n=2,3,4,5$. Also shown, for each configuration, is the circle of radius $X_{0}$ (see Eq. (38)), passing through its inflection points.

\subsection{Geometrics}

We are now in a position to give a purely geometrical construction of the equilibria. Indeed, starting from $X^{2}=h^{2}+p^{2}$ and using (37), we find

$$
X^{2}-X_{0}^{2}=4 \sigma^{-1} K, \quad X_{0} \equiv \sigma^{-1} \sqrt{8 E+\mu^{2}},
$$

where, in the derivation, use was made of the first integral, Eq. (9). This remarkable formula expresses the embedding completely in terms of $K(\ell)$. We emphasize that the shape is obtained directly from $K$, given $(\sigma, \mu, E)$, without any integration. Note that both the relations (37) follow by taking derivatives with respect to arclength of (38). It follows from (38) that the moment of inertia $\mathcal{I}$ of the loop around an axis perpendicular to its plane and passing through its center, is given by

$$
\mathcal{I}=L X_{0}^{2}+8 \pi \sigma^{-1} .
$$




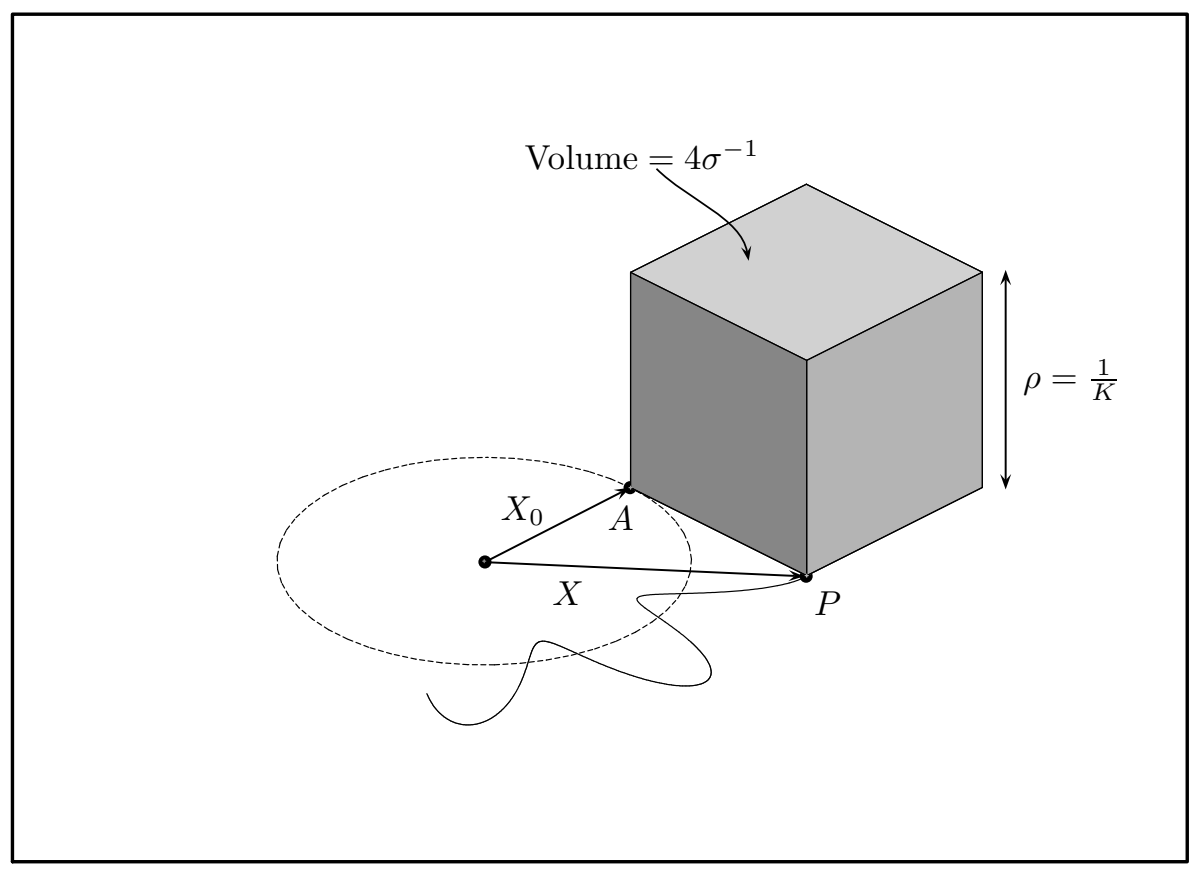

Figure 10: Geometrical construction of the equilibrium curve. The parallelepiped has square base with side $A P$ and volume $4 \sigma^{-1}$. Its height $\rho$ gives the radius of curvature at $P$. The part of the curve lying in the interior of the circle is constructed similarly.

To construct geometrically the equilibrium curve (for given $\sigma, \mu, E$ ), we draw a circle with radius $X_{0}$ and from a point $P$ outside it, bring the tangent $P A$ to the circle (see Fig. 10). Taking as base the square of this tangent, we construct a parallellepiped with volume $4 \sigma^{-1}$, the height of which is the radius of curvature of the equilibrium curve at $P$ - this follows from (38), written in the form $\left(X^{2}-X_{0}^{2}\right) \rho=4 \sigma^{-1}$, where $\rho$ is the radius of curvature at $P$. To achieve closure of the resulting curve, one has to start with a particular slope, given by either of (37). In this way, one constructs the part of the curve lying outside the circle (the latter intersects the curve at its inflection points, if any) - the interior part, present only if the ficticious particle reaches into the negative $K$ region, is constructed in a similar manner.

Referring back to our expression for the force $\vec{T}(\ell)$, Eq. (19), we realize that (37) implies that $\vec{T}(\ell)$ is orthogonal to $\vec{X}(\ell)$ while $T(\ell)=\sigma X(\ell)$, i.e.,

$$
\vec{T}(\ell)=\sigma(\hat{n} \times \hat{t}) \times \vec{X}(\ell), \quad T_{n}=-\sigma p, \quad T_{t}=\sigma h
$$

with $\vec{T}(\ell)$ the force on the part of the loop pointed to by $\hat{t}$. Notice that $\vec{T}$ is generally compressing but changes nevertheless to true tension at the points were $\vec{X}$ is tangential to the loop. Also, $p$ has acquired a direct physical interpretation as a consequence of (40): $\sigma p d \ell$ is just the torque, w.r.t. the origin, of the force due to the pressure on a segment of the loop with length $d \ell$. Then the vanishing of the total torque on the loop is guaranteed by the fact that $p$ is a derivative. In fact, one may derive a compact and rather 
pleasing formula for the torque due to pressure on any segment of the loop, like the one defined by $A, B$ in the sketch of an $n=4$ configuration of Fig. 11 .

Suppose we take $A$ as reference point, then (37) is valid with $\vec{a}$ connecting $A$ with the center $\mathcal{O}$ of the loop. We get

$$
\tau_{A B}^{(A)}=\sigma \int_{A}^{B} d \ell p=\sigma \int_{A}^{B} d \ell\left(2 \sigma^{-1} K^{\prime}+a_{t}\right)=2\left(K_{B}-K_{A}\right)+\sigma \vec{a} \cdot \int_{A}^{B} \overrightarrow{d \ell}
$$

i.e.,

$$
\tau_{A B}^{(A)}=2\left(K_{B}-K_{A}\right)+\sigma \vec{a} \cdot \overrightarrow{A B} .
$$

Moving the reference point simply moves one endpoint of $\vec{a}$. For $\vec{a}=0$ (torque w.r.t. $\mathcal{O}), \tau_{A B}^{(O)}=2\left(K_{B}-K_{A}\right)$.

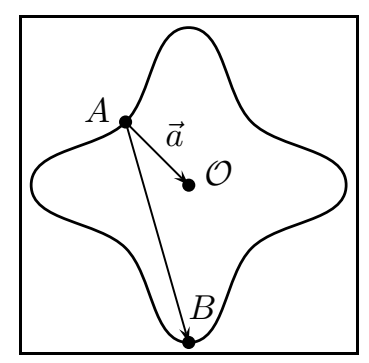

Figure 11: Computing the torque due to pressure on the arc $A B$

\subsection{Self-intersections}

Given that some potential applications of our model exclude self-intersecting configurations, we look now for sufficient conditions for non-self-intersection. We restrict our attention to self-intersections that can be reached by a continuous deformation of non-self-intersecting configurations. In other words, we consider a one-parameter family of configurations $\vec{X}(\ell, t), t \in[0,1]$, continuous in $t$, such that, for every $t$, the corresponding curvature satisfies (7) and we take $\vec{X}(\ell, 0), \vec{X}(\ell, 1)$ to be non-self-intersecting and self-intersecting respectively. Then, we observe that as $t$ varies from 0 to 1 , one necessarily encounters a "kiss": $\bigcap_{A}$ The position vector $\vec{X}_{A}$ is along the axis of symmetry of the lobe and tangential to the loop at $A$. The third of (40) then shows that the force at $A$ is normal to the loop, while the second of (40) gives its magnitude as

$$
T_{A}=\sigma X_{A} .
$$

The tangency of $\vec{X}$ at $A$ implies that $h_{A}=0$ and hence, using (37) once more, we get (assuming that $K$ is negative at a kiss $\left.\mathbb{1}^{2}\right)$

$$
K_{A}=-\sqrt{\mu}
$$

for all kisses, regardless of the order of the configuration. We conclude that a sufficient condition for non-self-intersection (of the type defined above), is $\mu<0$. (43) gives $K^{\prime 2}{ }_{A}$ in terms of $\sigma, \mu, E$

$$
{K^{\prime}}_{A}^{2}=2\left(E+\frac{1}{8} \mu^{2}-\frac{1}{2} \sigma \sqrt{\mu}\right) \text {. }
$$

On the other hand, from (38) we find that

$$
X_{A}^{2}=\sigma^{-2}\left(8 E+\mu^{2}\right)-4 \sigma^{-1} \sqrt{\mu} .
$$

The tangency of $X_{A}$ though means that $2 \sigma^{-1} K_{A}^{\prime}=p_{A}=X_{A}$ and the above two equations then give (setting $\sigma=1$ )

$$
E^{2}+\frac{1}{4}\left(\mu^{2}-4 \sqrt{\mu}-\frac{1}{2}\right) E+\frac{1}{64}\left(\mu^{2}-4 \sqrt{\mu}\right)\left(\mu^{2}-4 \sqrt{\mu}-1\right)=0,
$$

\footnotetext{
${ }^{4}$ This assumption is true for all configurations we have studied numerically.
} 
with roots

$$
E_{1}=\frac{1}{2} \sqrt{\mu}-\frac{1}{8} \mu^{2}+\frac{1}{8}, \quad E_{2}=\frac{1}{2} \sqrt{\mu}-\frac{1}{8} \mu^{2} .
$$

For the particular case $n=2, K_{A}^{\prime}=0$ and we get $E=E_{2}$. We have seen in Sec. 3 that the relation $\Theta_{0}=$ $2 \pi / n$ defines a curve in the $(\mu, E)$-plane, consisting of all parameter pairs giving rise to a configuration of order $n$. The intersection of that curve with the ones just written above, consists of the points $(\mu, E)$ giving rise to a kissing configuration of order $n$.

Consider how (40), (41) guarantee equilibrium in some particular examples. First, look at an even- $n$ configuration, say, $n=2: A \bigcirc B$ The total force due to the pressure, pushing together the two halves in the sketch, is $\sigma|\overrightarrow{A B}|$. (40) says that the tension at $A, B$ (purely tangential, compressing) is $\sigma|\overrightarrow{A B}| / 2$, thus leaving each half at rest. As a second example, consider the lobe defined by a selfintersection: $\gamma_{A}$ The total force due to the pressure on the lobe is zero, and (40) says that the forces from the rest of the loop, on the two ends of the lobe that meet at $A$, are opposite (with direction so as to keep the lobe closed). As a further check on our results, one can verify the balancing of torques using (41) on, say, the right half of a kiss.

\subsection{Connecting equilibria}

There is more to be derived from (38). Taking $\delta_{\epsilon}$ on both sides, we find

$$
D E_{2}^{(a)}(\epsilon) \equiv 2 \epsilon^{\prime \prime}+\left(3 K^{2}-\mu\right) \epsilon=\frac{\sigma}{2} \delta_{\epsilon} X_{0}^{2}-2 K \sigma^{-1} \delta_{\epsilon} \sigma,
$$

a considerable improvement over (29) 5 . Moreover, by varying the first of (37) we also find

$$
D E_{2}^{(b)}(\epsilon) \equiv 2 K \epsilon^{\prime \prime}-2 K^{\prime} \epsilon^{\prime}+\left(\sigma+2 K^{3}\right) \epsilon=-\sigma^{-1}\left(K^{2}-\mu\right) \delta_{\epsilon} \sigma-\delta_{\epsilon} \mu .
$$

Comparison with (48) leads to a first order equation for $\epsilon$

$$
D E_{1}(\epsilon) \equiv 4 K^{\prime} \epsilon^{\prime}-4 K^{\prime \prime} \epsilon=c_{2} K^{2}+c_{1} K+c_{0},
$$

where the constants $c_{i}$ are given by

$$
c_{2} \equiv-2 \sigma^{-1} \delta_{\epsilon} \sigma, \quad c_{1} \equiv \sigma \delta_{\epsilon} X_{0}^{2}, \quad c_{0} \equiv 2 \delta_{\epsilon} \mu-2 \mu \sigma^{-1} \delta_{\epsilon} \sigma .
$$

Noting that $\frac{d}{d K}=\left(K^{\prime}\right)^{-1} \frac{d}{d \ell},(50)$ can be written in the form

$$
8(E-V) \dot{\epsilon}+4 \dot{V} \epsilon=c_{2} K^{2}+c_{1} K+c_{0},
$$

where the dot denotes differentiation w.r.t. $K$ and $V=V(K)$. This latter equation is readily integrated to give

$$
\epsilon=\frac{1}{4} K^{\prime} \int d K \frac{c_{2} K^{2}+c_{1} K+c_{0}}{(2(E-V))^{3 / 2}}
$$

One may add an arbitrary amount of $K^{\prime}$ (rotation) to this (but not $a_{n}$ or $a_{t}$, since these move the origin). To get particular solutions from (53), we need to specify the direction of the deformation in the $(\sigma, \mu)$ plane, i.e., the ratio $\delta_{\epsilon} \mu / \delta_{\epsilon} \sigma$. It will prove convenient for our further analysis of (53), to reparameterize the $(\sigma, \mu)$-plane introducing new coordinates $(\lambda, \xi)$ via

$$
\lambda(\sigma, \mu)=\sigma^{-1 / 3}, \quad \xi(\sigma, \mu)=\sigma^{-2 / 3} \mu .
$$

\footnotetext{
${ }^{5}$ Notice, however, that (48) is only valid when the center of the loop is at the origin.
} 
A point $P$ with coordinates $(\sigma, \mu) \neq(0,0)$ lies on a unique scaling orbit, which can be specified by the $\mu$-coordinate of its point of intersection $P^{\prime}$ with the $\sigma=1$ line, this is the value of $\xi$ for $P$. One can get now from $P^{\prime}$ to $P$ by scaling by $\lambda$ (see Fig. 12). The obvious advantage of these new coordinates is that the scaling orbits are constant- $\xi$ lines. The dependence on $\lambda$ of an arbitrary quantity $S(\lambda, \xi)$, with length dimension $q$, is $S(\lambda, \xi)=\lambda^{q} \tilde{S}(\xi)$, where we denote by a tilde the remaining function of $\xi$. It follows that $\frac{\partial S}{\partial \lambda}=q \lambda^{-1} S$ so that $\delta_{\epsilon} S=q \lambda^{-1} S \delta_{\epsilon} \lambda+\lambda^{q} \tilde{S}^{\prime} \delta_{\epsilon} \xi$, the prime denoting here differentiation w.r.t. $\xi$.

We verify that (53) gives $\epsilon \sim h$ for scaling. Eq. (51), written in terms of $(\lambda, \xi)$, gives

$$
\begin{aligned}
& c_{2}=6 \lambda^{-1} \delta_{\epsilon} \lambda \\
& c_{1}=\lambda^{-2}\left(16 \tilde{E}+2 \xi^{2}\right) \delta_{\epsilon} \lambda+\lambda^{-1}\left(8 \tilde{E}^{\prime}+2 \xi\right) \delta_{\epsilon} \xi \\
& c_{0}=2 \lambda^{-3} \xi \delta_{\epsilon} \lambda+2 \lambda^{-2} \delta_{\epsilon} \xi,
\end{aligned}
$$

where $E(\lambda, \xi)=\lambda^{-4} \tilde{E}(\xi)$ is the energy that guarantees closure of some particular configuration. An increment $\delta_{\epsilon} \lambda$ corresponds to scaling by a factor $1+\delta_{\epsilon} \lambda / \lambda$, hence the corresponding $\epsilon$ should be $\epsilon=\left(\delta_{\epsilon} \lambda / \lambda\right) h$. Putting $\delta_{\epsilon} \xi=0$ in (56) one determines the $c_{i}$ for pure scaling, then computing $d / d K\left(\left(K^{2}-\mu\right) / K^{\prime}\right)$ one finds that the $K^{5}$ and $K^{3}$ terms in the numerator cancel and one recovers the integrand in (53) with just the right $c$ 's.

Integrating the first of (37) w.r.t. $d \ell$, and using

$$
A=\frac{1}{2} \int d \ell h,
$$

we get

$$
F=\mu L+2 \sigma A
$$

from which

$$
\delta_{\epsilon} F=L \delta_{\epsilon} \mu+\mu \delta_{\epsilon} L+2 A \delta_{\epsilon} \sigma+2 \sigma \delta_{\epsilon} A
$$

follows. On the other hand, direct variation of $F$, Eq. (2), gives

$$
\delta_{\epsilon} F=-\mu \delta_{\epsilon} L-\sigma \delta_{\epsilon} A \text {. }
$$

Comparing with $(58)$ we find

$$
L \delta_{\epsilon} \mu+2 A \delta_{\epsilon} \sigma+3 \sigma \delta_{\epsilon} A+2 \mu \delta_{\epsilon} L=0 .
$$

For a length-preserving deformation, $\delta_{\epsilon} L=0$, and (60) reduces to

$$
L \delta_{\epsilon} \mu+2 A \delta_{\epsilon} \sigma+3 \sigma \delta_{\epsilon} A=0 .
$$

We note in passing that the various differential operators we have defined above are related in the following way

$$
\begin{aligned}
{\left[D E_{1}(\epsilon)\right]^{\prime} } & =2 K^{\prime} D E_{2}{ }^{(a)}(\epsilon), \\
K\left[D E_{1}(\epsilon)\right]^{\prime} & =2 K^{\prime} D E_{2}{ }^{(b)}(\epsilon)+K^{\prime} D E_{1}(\epsilon), \\
D E_{3}(\epsilon) & =K^{\prime}\left[D E_{2}{ }^{(a)}(\epsilon)\right]^{\prime}-K^{\prime \prime} D E_{2}{ }^{(a)}(\epsilon)-\frac{1}{4}\left(K^{2}-\mu\right) D E_{1}(\epsilon), \\
K^{\prime} D E_{4}(\epsilon) & =2\left[D E_{3}(\epsilon)\right]^{\prime} .
\end{aligned}
$$

It follows that any perturbation that satisfies Eq. (50) will necessarily satisfy all the higher order ones. 


\subsection{Perturbing the circle}

Referring to our cylinder model of the loop (see Sec. 2.5), we consider here the following thought experiment: we start with the loop "filled" to capacity, i.e., in a circular configuration, and start deflating it by removing fluid from its interior while keeping its length fixed. We would like to follow the evolution of its shape, for distinct $n$ 's, and derive the limiting form of the bending energy $F$ as a function of the area $A$ near the circular extreme. Going beyond the cylinder model, we would also like to allow for self-intersections — what happens if one just keeps subtracting area?

Consider the following perturbation to a circle of radius $R$

$$
\rho(\theta)=R_{\alpha}(1+\alpha \sin (n \theta)), \quad \alpha \ll 1 .
$$

The line element and curvature in these coordinates are

$$
d \ell=\sqrt{\rho^{2}+\dot{\rho}^{2}} d \theta, \quad K=\frac{\rho^{2}+2 \dot{\rho}^{2}-\rho \ddot{\rho}}{\left(\rho^{2}+\dot{\rho}^{2}\right)^{3 / 2}},
$$

where the dot here denotes differentiation w.r.t. $\theta$. Substituting (62) in the first of (63), integrating over $\theta$ and requiring the total length to be equal to the initial value $2 \pi R$, we find

$$
R_{\alpha}=R\left(1-\frac{n^{2} \alpha^{2}}{4}\right)+\mathcal{O}\left(\alpha^{3}\right)
$$

while for the curvature the second of $(63)$ gives

$$
K=\frac{1}{R}\left(1+\alpha\left(n^{2}-1\right) \sin (n \theta)\right)+\mathcal{O}\left(\alpha^{2}\right) .
$$

We substitute the above expression in the differential equation for $K$, Eq. (7), and demand that it be a solution to $\mathcal{O}(\alpha)$. The constant and $\mathcal{O}(\alpha)$ terms respectively give

$$
1-R^{2} \mu-R^{3} \sigma=0, \quad 2 n^{4}+\left(R^{2} \mu-5\right) n^{2}+3-R^{2} \mu=0 .
$$

Notice that the first relation is simply the statement $\dot{V}(1 / R)=0$ while the second also follows from (29) with $\epsilon$ as in (62). For this perturbation the differential equation cannot be satisfied to $\mathcal{O}\left(\alpha^{2}\right)$. From $(66)$ we get

$$
\sigma=\frac{2}{R^{3}}\left(n^{2}-1\right), \quad \mu=\frac{1}{R^{2}}\left(3-2 n^{2}\right) .
$$

During the deflating process, both $\sigma$ and $\mu$ will vary as functions of $A$. The point in the $\sigma-\mu$ plane corresponding to the configuration will trace out an orbit, starting at the above points (for each $n$ ), all of which lie on the line $R^{3} \sigma+R^{2} \mu=1$. For $F$ and $A$ we find

$$
F=\frac{2 \pi}{R}\left(1+\frac{1}{2}\left(n^{2}-1\right)^{2} \alpha^{2}\right)+\mathcal{O}\left(\alpha^{3}\right), \quad A=\pi R^{2}\left(1-\frac{1}{2}\left(n^{2}-1\right) \alpha^{2}\right)+\mathcal{O}\left(\alpha^{3}\right) .
$$

Notice that, near the circle,

$$
\frac{\delta F}{\delta A}=-\frac{2}{R^{3}}\left(n^{2}-1\right)=-\sigma
$$

in agreement with (59) (for $\delta L=0$ ). As $A$ keeps diminishing, our numerical analysis shows that the configurations start self-intersecting, giving rise to regions of negative area - this scenario is sketched in Fig. 13. Notice how the winding numbers $(+1$ for the little circles, -1 for the big one) add up correctly to match that of the circle to which this configuration can be continuously deformed. This observation points to a feature of the above $m=1$ limiting configurations already alluded to in the introduction: the big circle in the configuration of order $n$ actually winds around itself $n-1$ times. For example, to move 


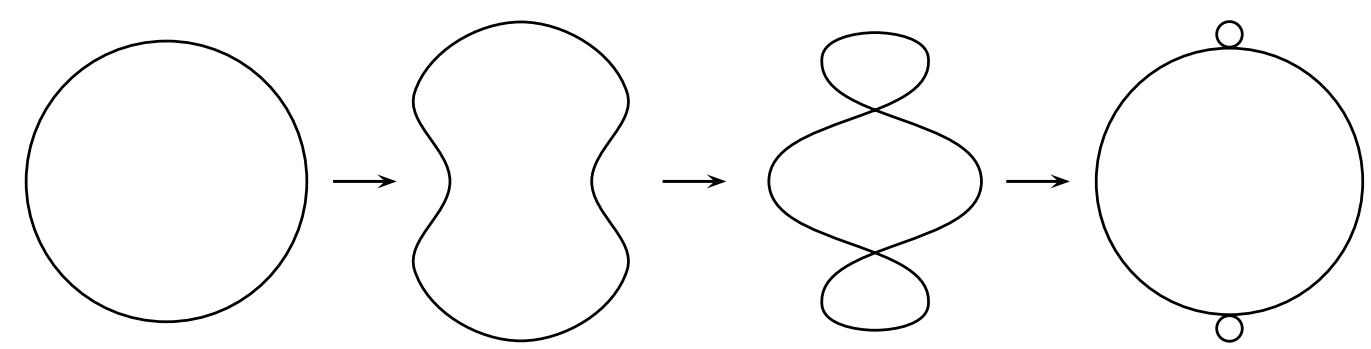

Figure 13: Evolution of a $n=2$ configuration under deflation (sketch). The central region in the third configuration, as well as the big circle in the fourth one, contribute negative area.

from one lobe to the next along the loop, in the $n=3$ configuration, one must travel an angle of $4 \pi / 3$, not $2 \pi / 3$ (a glance at Fig. 9.b reveals how this comes about). The limiting shape in this direction then is a circular one, with radius slightly less than $R /(n-1)$ and $n$ little circles attached to it. In this extreme, $F(A)$ is dominated by the little circles and assumes the limiting form

$$
F(A) \cong \frac{4 \pi n^{2}}{R} \frac{1}{1+(n-1) \frac{A}{\pi R^{2}}},
$$

where the area is counted with the appropriate multiplicity due to the winding (e.g., plus three times the area of the little circle, minus twice that of the big one, in the $n=3$ case). A sketch of $F(A)$ that interpolates between (68) and (69) is given in Fig. 14.

\section{Conclusions}

In this paper, we have studied the equilibrium configurations of elastic planar loops with constant area and constant length. The condition of closure of the loop gives a discrete spectrum of configurations, lying along several branches in parameter space. We focus on non self-intersecting loops that inflate to a circle when the enclosed area is increased, due to their relevance as toy model for two-dimensional membranes. For this branch, starting from analytical expressions in the relevant limit cases, we obtain a reliable sketch of the dependence of the energy on the area. The equation that determines equilibria connecting perturbations led us, rather unexpectedly, to the $\sigma$-identities (37), from which a novel geometrical construction of the (constrained) elastic loop followed. In turn, these identities permit the expression of an equilibrium connecting normal deformation in terms of the curvature and the appropriate perturbations of the parameters.

In future work, we plan to address the important issue of the stability of the equilibrium configurations. In light of the complicated structure of the fourth order differential operator appearing in (29), this appears to be a non-trivial task in the general case. It is indicative of the intrinsic complexity of the question that even the relatively simple case of the figure eight configuration requires the elaborate analysis of 12 .

Another interesting issue is the analysis of thermal fluctuations in this model, which would provide an analytical counterpart to the study of Ref. [16] of two-dimensional vesicles, using Monte Carlo techniques. In particular, there is a similarity between some of the configurations of Fig. 2 of the above work and the configuration of Fig. 8.a in this paper. It is also interesting to note that the shapes of Fig. 8 closely resemble a top view of the starfish vesicles of Ref. [28], which are almost planar. Finally, configurations similar to ours appear in 24], where our problem has been approached from a functional analytic point of view and important existence results have been derived, as well as in [8]. 


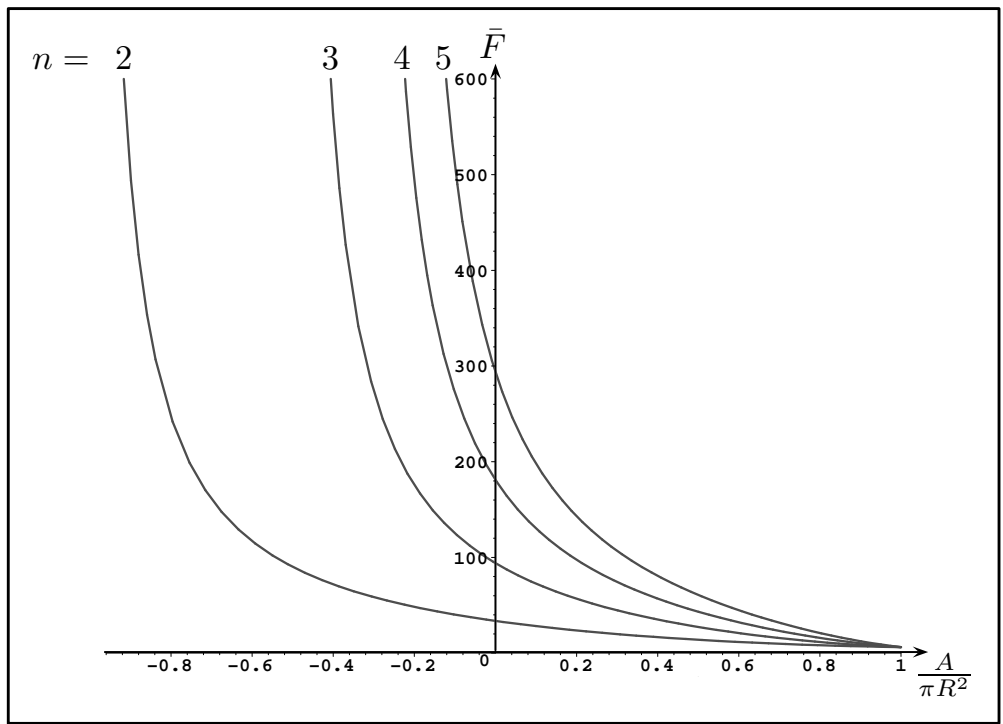

Figure 14: Bending energy $\bar{F}$ vs. area $A / \pi R^{2}$ for $n=2,3,4,5$ (interpolation). All curves touch at the point on the right, which corresponds to a circular configuration, giving rise to bifurcation. The vertical asymptotes are at $-\frac{1}{n-1}$.

After the completion of this paper, we set out to study its most natural extension: geometric models for loops in space. We became aware of a large body of literature that explores the interconnections among hierarchies of functionals of the geometry of a curve, their corresponding generators of rigid motion and integrable systems, such as the KdV equation. We have found Ref. [13] an excellent point of entry to the subject. We also realized that our $\sigma$-identities can be obtained by adapting the cylindrical coordinates used by Langer and Singer 14] in the analysis of buckled rings. We expect that these explorations, apart from their intrinsic interest, will contribute to a deeper understanding of two-dimensional membranes.

\section{Acknowledgements}

G.A. wishes to thank Gilberto Tavares for technical assistance and CONACyT for a graduate fellowship. R.C. was supported by CONACyT grant 32187-E. G.A., C.C. and J.G. were supported by CONACyT grant 32307-E and DGAPA-UNAM grant IN119792.

\section{A Some Useful Formulae}

Under an infinitesimal deformation of the loop along the normal, $\vec{X} \rightarrow \vec{X}+\delta_{\epsilon} \vec{X} \equiv \vec{X}+\epsilon(\ell) \hat{n}$, we find

$$
\begin{array}{rlrl}
\delta_{\epsilon} d \ell=K \epsilon d \ell, & \delta_{\epsilon} \hat{t}=\epsilon^{\prime} \hat{n}, & \delta_{\epsilon} K=-\epsilon^{\prime \prime}-K^{2} \epsilon \\
\delta_{\epsilon} \frac{d}{d \ell}=-K \epsilon \frac{d}{d \ell}, & \delta_{\epsilon} \hat{n}=-\epsilon^{\prime} \hat{t}, & \delta_{\epsilon} K^{\prime}=-\epsilon^{\prime \prime \prime}-K^{2} \epsilon^{\prime}-3 K K^{\prime} \epsilon \\
\delta_{\epsilon} h=\epsilon-p \epsilon^{\prime}, & \delta_{\epsilon} p=\epsilon^{\prime} h, & \delta_{\epsilon} K^{\prime \prime}=-\epsilon^{\prime \prime \prime \prime}-K^{2} \epsilon^{\prime \prime}-5 K K^{\prime} \epsilon^{\prime} \\
& & &
\end{array}
$$

(we have used the e.o.m and the first integral, Eqs. (7) and (9), to express $K^{\prime \prime}$ and $\left(K^{\prime}\right)^{2}$ in terms of $K$ ). 
For the derivatives of $h, p, K$ we find

$$
\begin{aligned}
h^{\prime} & =K p \\
h^{\prime \prime} & =-K^{2} h+K^{\prime} p+k \\
h^{\prime \prime \prime} & =-3 K K^{\prime} h+\left(-\frac{3}{2} K^{3}+\frac{\mu}{2} K+\frac{\sigma}{2}\right) p+2 K^{\prime} \\
h^{\prime \prime \prime \prime} & =\left(-6 E+\frac{15}{4} K^{4}-\frac{7}{2} \mu K^{2}-5 \sigma K\right) h+\left(-\frac{15}{2} K^{2} K^{\prime}+\frac{\sigma}{2} K^{\prime}\right) p-\frac{5}{2} K^{3}+\frac{3}{2} \mu K+\frac{3}{2} \sigma \\
p^{\prime} & =1-K h \\
p^{\prime \prime} & =-K^{\prime} h-K^{2} p \\
p^{\prime \prime \prime} & =\left(\frac{3}{2} K^{3}-\frac{\mu}{2} K-\frac{\sigma}{2}\right) h-3 K K^{\prime} p-K^{2} \\
p^{\prime \prime \prime \prime} & =\left(\frac{15}{2} K^{2} K^{\prime}-\frac{\mu}{2} K^{\prime}\right) h+\left(\frac{15}{4} K^{4}-\frac{7}{2} \mu K^{2}-5 \sigma K-6 E\right) p-5 K K^{\prime} \\
K^{\prime} & =\sqrt{2}\left(E-\frac{1}{8} K^{4}+\frac{\mu}{4} K^{2}+\frac{\sigma}{2} K\right)^{1 / 2} \\
K^{\prime \prime} & =\frac{1}{2}\left(-K^{3}+\mu K+\sigma\right) \\
K^{\prime \prime \prime} & =-\frac{3}{2} K^{2} K^{\prime}+\frac{\mu}{2} K^{\prime} \\
K^{\prime \prime \prime \prime} & =\frac{3}{2} K^{5}-\frac{10}{4} \mu K^{3}-\frac{15}{4} \sigma K^{2}+\left(\frac{\mu^{2}}{4}-6 E\right) K+\frac{\mu \sigma}{4}
\end{aligned}
$$

\section{B Averages}

Consider the quantities $W_{n}$, defined by

$$
W_{n}=\int_{0}^{L_{0}} K^{n} d \ell=2 \int_{K_{\min }}^{K_{\max }} K^{n} \frac{d K}{K^{\prime}} .
$$

$L_{0}$ here is the length along the loop corresponding to one full oscillation of the particle (we will use $F_{0}$ later with a similar meaning). $W_{n}$ is then (proportional to) the average of $K^{n}$ along the loop. Starting from

$$
\int_{K_{\min }}^{K_{\max }} \frac{d}{d K}\left(K^{n} K^{\prime}\right)=0
$$

one may derive the recursion relation [1]

$$
-\frac{1}{8}(n+2) W_{n+3}+\frac{\mu}{4}(n+1) W_{n+1}+\frac{\sigma}{4}(2 n+1) W_{n}+E n W_{n-1}=0, \quad n=0,1,2, \ldots,
$$

which permits, in principle, the calculation of the average of any power series in $K$, for a given equilibrium configuration. In particular,

$$
W_{3}=\mu W_{1}+\sigma W_{0}, \quad W_{4}=\frac{4}{3} W_{2}+2 \sigma W_{1}+\frac{8}{3} E W_{0} .
$$

Referring back to Fig. 1, we notice that the area $S$ enclosed by the orbit of the phase point, for some given $\sigma, \mu, E$, is given by

$$
S(\sigma, \mu, E)=2 \int_{K_{\min }}^{K_{\max }} K^{\prime} d K
$$


Differentiating w.r.t. $\sigma$ and using the fact that the integrand vanishes at the endpoints, we find

$$
\Theta_{0}=2 \frac{\partial S}{\partial \sigma} .
$$

Length and bending energy per full oscillation also follow by simple differentiation

$$
L_{0}=\frac{\partial S}{\partial E}, \quad F_{0}=4 \frac{\partial S}{\partial \mu} .
$$

Notice that these quantities are defined whether or not the closure condition is satisfied, i.e., they are functions of the three independent variables $\sigma, \mu, E$. Combining (74) and (73) above, we get

$$
S=\frac{4}{3} E L+\frac{1}{6} \mu F+\frac{1}{2} \sigma \Theta .
$$

One may regard $L_{0}, \Theta_{0}$ and $F_{0}$ as new coordinates in the space of (not necessarily closed) configurations

- the change of coordinates is non-singular, except for some special points, and is given by a Legendre transform. Then the closure condition, Eq. (11), restricts to the configurations that lie on the $L_{0}-F_{0}$ plane, with $\Theta_{0}=2 \pi / n$.

\section{References}

[1] M. Abramowitz and I.A. Stegun. Handbook of Mathematical Functions. Dover Publications, New York, 1965.

[2] J. Ambjorn, B. Durhuus, and T. Jonsson. Quantum Geometry: A statistical field approach. Cambridge University Press, 1997.

[3] C. Bouchiat and M. Mezard. Elasticity Model of a Supercoiled DNA Molecule. Phys. Rev. Lett., 80:1156-1159, 1998.

[4] R. Bryant and P. Griffiths. Reduction for Constrained Variational Problems and $\int \frac{\kappa^{2}}{2} d s$. Amer. Journ. of Math., 108:525-570, 1986.

[5] P.F. Byrd and M.D. Friedman. Handbook of Elliptic Integrals for Engineers and Physicists. Springer Verlag, Berlin, 1954.

[6] H.J. Deuling and W. Helfrich. The Curvature Elasticity of Fluid Membranes: a Catalogue of Vesicle Shapes. J. Phys. (Paris), 37:1335-1345, 1976.

[7] M. do Carmo. Differential Geometry of Curves and Surfaces. Prentice Hall, 1976.

[8] Y. Fukumoto. Stationary Configurations of a Vortex Filament in Background Flows. Proc. R. Soc. Lond. A, 453:1205-1232, 1997.

[9] M. Giaquinta and S. Hildebrandt. Calculus of Variations I. Springer Verlag, 1996.

[10] P. A. Griffiths. Exterior Differential Systems and the Calculus of Variations. Birkhaüser, Boston, 1983.

[11] W. Helfrich. Elastic Properties of Lipid Bilayers: Theory and Possible Experiments. Z. Naturforsch., C28:693-703, 1973.

[12] T. Ivey and D. Singer. Knot Types, Homotopies and Stability of Closed Elastic Rods. math.DG/9901131. 
[13] J. Langer. Recursion in Curve Geometry. New York Journ. of Math., 5:25-51, 1999.

[14] J. Langer and D. Singer. Knotted Elastic Curves in $R^{3}$. J. London Math. Soc., 30:512-520, 1984.

[15] J. Langer and D. Singer. The Total Squared Curvature of Closed Curves. Journ. Diff. Geom., 20:1-22, 1984.

[16] S. Leibler, R. R. P. Singh, and M. E. Fisher. Thermodynamic Behavior of Two-dimensional Vesicles. Phys. Rev. Lett., 59:1989-1992, 1987.

[17] J.F. Marko and E.D. Siggia. Bending and Twisting Elasticity of DNA. Macromolecules, 27:981, 1994. Streching DNA. ibid. 28:8759 (1995).

[18] Z.C. Ou-Yang and W. Helfrich. Instability and Deformation of a Spherical Vesicle by Pressure. Phys. Rev. Lett., 59:2486-2488, 1987.

[19] Z.C. Ou-Yang and W. Helfrich. Bending Energy of Vesicle Membranes: General Expressions for the First, Second and Third Variation of the Shape Energy and Applications to Spheres and Cylinders. Phys. Rev., A39:5280-5288, 1989.

[20] L. Peliti. Amphiphilic Membranes. In F. David, P. Ginsparg, and J. Zinn-Justin, editors, Fluctuating Geometries in Statistical Physics and Field Theory (Les Houches 1994). Elsevier Science B.V., 1996.

[21] S.A. Safran. Statistical Physics of Surfaces, Interfaces, and Membranes. Reading UK, Addison Wesley, 1994.

[22] U. Seifert. Configurations of Fluid Membranes and Vesicles. Adv. in Phys., 46:13-137, 1997.

[23] S. Svetina and B. Zeks. Memebrane Bending Energy and Shape Determination of Phospholipid Vesicles and Red Blood Cells. Euro. Biophys. J., 17:101-111, 1989.

[24] I. Tadjbakhsh and F. Odeh. Equilibrium States of Elastic Rings. J. of Math. Anal. Appl., 18:59-74, 1967.

[25] C. Truesdell. The Influence of Elasticity on Analysis: the Classical Heritage. Bull. Amer. Math. Soc., 9:293-310, 1983.

[26] T.J. Willmore. Total Curvature in Riemannian Geometry. Ellis Horwood, Chichester, England, 1982.

[27] T.J. Willmore. Curves, volume 1 of Handbook of Differential Geometry, chapter 11, pages 997-1023. Elsevier Science B.V., 2000.

[28] W. Wintz, H. H. Dobereiner, and U. Seifert. Starfish Vesicles. Europhys. Lett., 33:403-408, 1996. 